\title{
Synthesis of sterically hindered 3-(azolyl)pyridines
}

\author{
Sergey M. Lukyanov, * Igor V. Bliznets, and Sergey V. Shorshnev \\ ChemBridge Corporation, Malaya Pirogovskaya 1, 119435 Moscow, Russian Federation \\ E-mail: semiluk@gmail.com
}

Dedicated to Professor Alexander F. Pozharskii on his $\mathbf{7 0}^{\text {th }}$ birthday

\begin{abstract}
Sterically hindered 2,4-disubstituted 3-(1,2,4-oxadiazol-3-yl)-, 3-(imidazol-2-yl)- and 3-(thiazol-2yl)pyridines were synthesized from the corresponding nicotinonitriles via amidoximes, amidines and thioamides, respectively. $N$-Alkyl- and $N$-arylamidines were prepared directly from nicotinonitriles using microwave technology. The series of 3-(azolyl)pyridines form a combinatorial library of heterocyclic derivatives of nicotinic acid and were examined by prognostic software PASS.
\end{abstract}

Keywords: Nicotinonitriles, 1,2,4-oxadiazoles, imidazoles, thiazoles, microwave irradiation

\section{Introduction}

One of the most promising strategies for searching for new pharmaceuticals and improving the properties of known ones is a replacement of metabolically unstable functional groups with bioisosteric five-membered heterocyclic rings. ${ }^{1,2}$ The best-known azole that is used as a metabolism-resistant surrogate for the carboxylate group is 5-substituted tetrazole, since they both possess comparable acidity and size. ${ }^{3}$ Nicotinic acid and its derivatives can be very often found among the carboxylic acids investigated with respect to such replacements. ${ }^{2,4}$ Thus, 3-(5tetrazolyl)pyridines were reported as a new class of lipolysis inhibitors ${ }^{5}$ and bioisosteres of arecoline. $^{2}$

At the same time, other azoles can also serve as the isosteric equivalents for labile functional groups in the derivatives of nicotinic acid. Thus, 3-(1,3,4-oxadiazol-2-yl)pyridines show antimicrobial and antifungal activity ${ }^{6}$ and were tested as nonpeptidic inhibitors of human neutrophil elastase. ${ }^{7}$ The corresponding derivatives of 1,2,4-oxadiazole displayed high affinity and efficacy as muscarinic agonists. ${ }^{8}$ An antimycobacterial ${ }^{9}$ as well as an anthelmintic ${ }^{10}$ activity of pyridines substituted with 1,2,4-oxadiazoles as isosteres of nicotinic acid was described. 3-(Imidazol-2yl)pyridines are known as inhibitors of xanthine oxidase, ${ }^{11}$ nonsteroidal antiinflammatory agents, ${ }^{12}$ potent and selective NPY5 receptor antagonists ${ }^{13}$ as well as being tested as KDR kinase inhibitors. ${ }^{14}$ 3-(Thiazol-2-yl)pyridines are known as inhibitors of superoxide production by human neutrophils, ${ }^{15}$ 
selective cyclooxygenase- 2 inhibitors ${ }^{16}$ and effective inhibitors of human cytochrome P-450 (CYP2A6), the major nicotine metabolizing enzyme; ${ }^{17}$ they also displayed antifungal and antibacterial ${ }^{18}$ as well as antitubercular activity; $;{ }^{19}$ besides, these compounds were used as building block for synthesis of berninamycinic acid ${ }^{20}$ and heterocyclic core of micrococcin $\mathrm{P} 1,{ }^{21}$ preparation of the central heterocyclic skeleton of antibiotic A10255, ${ }^{22}$ macrocyclic antibiotic GE $2270 \mathrm{~A}^{23}$ and other various thiostrepton-type macrocyclic antibiotics. ${ }^{24}$ On the other hand, it was found that pyridine derivatives bearing a sterically hindered tetrazole unit at position 3 show some very interesting pharmacological properties. ${ }^{25}$

In view of above, it would be interesting to create a combinatorial library consisting of sterically hindered 3-(azolyl)pyridines 2-6 as derivatives of nicotinic acid (Figure 1). Such combinatorial library would be also of interest since only two examples of 2,4,5-trisubstituted 3-(azolyl)pyridines [namely, 3-(1,2,4-oxadiazol-3-yl)pyridines, structure 4] were found in the literature. ${ }^{26}$ It should be noted that these two compounds were identified as mixtures (purity 60\%) prepared with low yields $(25 \%)$.

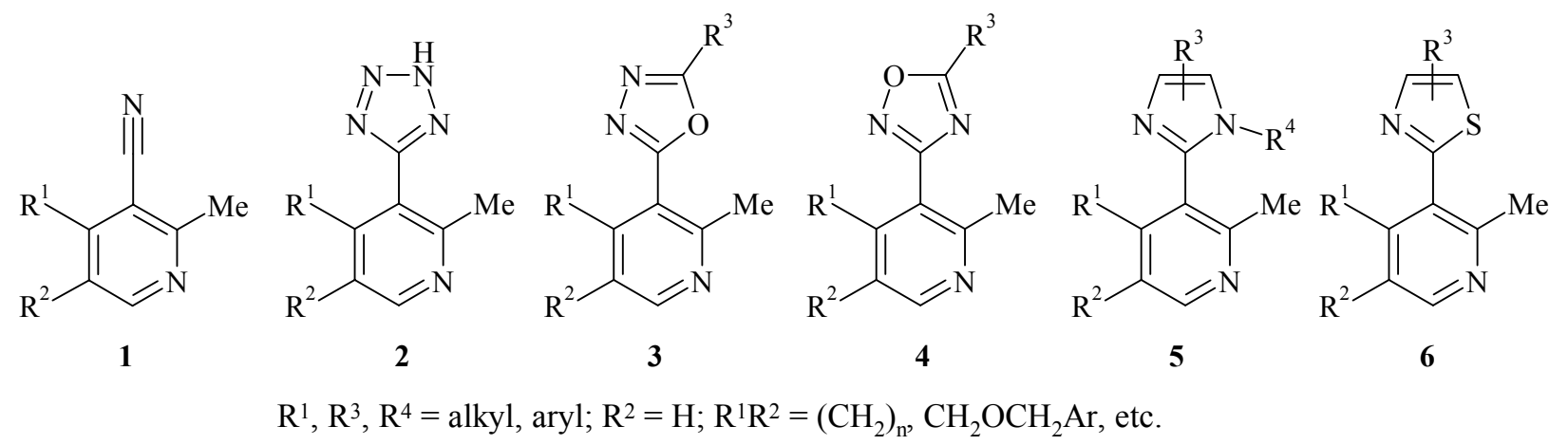

Figure 1. Nicotinonitriles 1 and 3-(azolyl)pyridines 2-6 as components of combinatorial library.

Recently, we have reported the microwave-assisted synthesis of 3-(5-tetrazolyl)pyridines $\mathbf{2}$ and their fused polycyclic derivatives from sterically hindered nicotinonitriles $1 .^{27,28}$ The synthesis of 3(1,3,4-oxadiazol-2-yl)pyridines $\mathbf{3}$ from the 3-(5-tetrazolyl)pyridines $\mathbf{2}$ was also described in one of these papers. ${ }^{27}$ Herein, we wish to report the synthesis of 2,3,4-tri- and 2,3,4,5-tetrasubstituted pyridines bearing 1,2,4-oxadiazol-3-yl (4), imidazol-2-yl (5), and thiazol-2-yl (6) moieties at position 3 as well as methyl group at position 2. It should be noted that the 2,3-di- and 2,4,5trisubstituted nicotinonitriles $\mathbf{1}$ had to be used as starting compounds common for the entire combinatorial library.

\section{Results and Discussion}

Two syntheses of 1,2,4-oxadiazoles $\mathbf{1 2}$ from nitriles $\mathbf{7}$ are widely known. ${ }^{29}$ The first can be accomplished via the imidates $\mathbf{8},{ }^{30}$ and the second approach is the condensation of amidoximes $\mathbf{1 0}$ with carboxylic acid derivatives (anhydrides, chlorides) followed by final ring closure (Scheme 1). The acylated intermediates $\mathbf{9 , 1 1}$ were mentioned as isolable compounds for both reaction routes. ${ }^{29}$ 


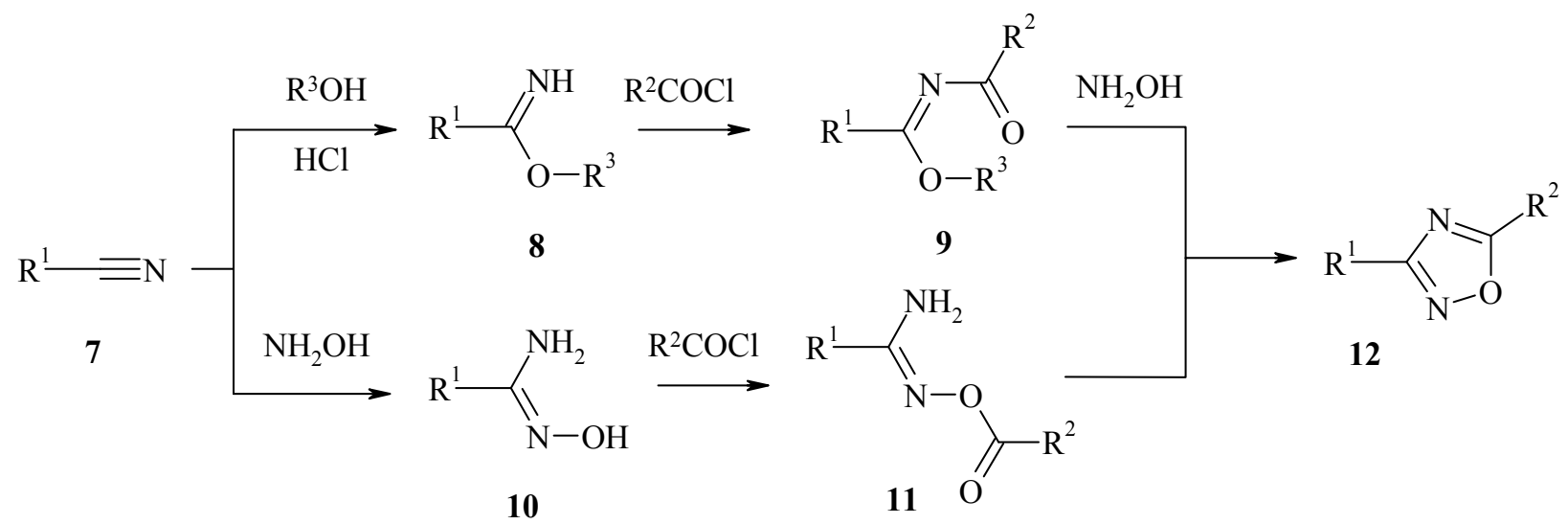

Scheme 1. Generalized synthesis of 1,2,4-oxadiazoles 12 from nitriles 7.

It was insisted in many related publications that the both imidates $\mathbf{8}$ and amidoximes $\mathbf{1 0}$ can be easily prepared using facile procedures $9,10,30,31$ including conditions of parallel synthesis. ${ }^{8 \mathrm{~d}, 26,32}$ It should be noted that aromatic nitriles described in many of these papers had no substituents alongside of cyano group. However, all our attempts to prepare the imidates $\mathbf{8}$ from nicotinonitriles 1 failed. It was not surprising to us, since many reactions of nitrile groups are very susceptible to steric hindrance (for example, the Pinner reaction). ${ }^{33}$ Further, the same result was obtained when we attempted to synthesize the amidoximes $\mathbf{1 0}$ from nicotinonitriles $\mathbf{1}$ using some conventional procedures (interaction of nitriles with $\mathrm{NH}_{2} \mathrm{OH} \cdot \mathrm{HCl}$ in the presence of various bases). Indeed, a dependence of this reaction on sterical circumstances was also reported. ${ }^{31 \mathrm{~d}}$

Now, we have found that the sterically hindered nicotinonitriles $\mathbf{1}$ can be converted into desired amidoximes 13 by long-continued heating with 50\% aqueous solution of hydroxylamine (Scheme 2). The resultant amidoximes $\mathbf{1 3}$ were in many cases contaminated by corresponding amides 14 yields of which increased along with growth of volume of the substituent $\mathrm{R}^{1}$ at position 4 (Table 1). Finally, amide 14e turned out the sole reaction product for 4-tert-butylsubstituted nicotinonitrile $1 \mathrm{e}$. It was reported previously that such amide formation is a result of initial attack by the oxygen atom of hydroxylamine to cyano group but not due to hydrolysis of nitrile or amidoxime. ${ }^{34}$ We found that the "amidoxime: amide" ratio did not depend on ratios of starting reagents as well as temperature and reaction time. Unfortunately, our attempts to improve the yields of amidoximes $\mathbf{1 3}$ as well as to reduce the reaction time using microwave conditions turned out also to be inefficient.<smiles>[R]c1cnc(C)c(C#N)c1[R]</smiles>

1a-e

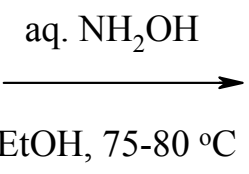

EtOH, $75-80{ }^{\circ} \mathrm{C}$<smiles>[R]c1cnc(C)c(C(N)=NO)c1[R]</smiles>

13a-d<smiles>[R]c1cnc(C)c(C(N)=O)c1[R]</smiles>

14b-e

Scheme 2. Synthesis of sterically hindered amidoximes 13. 
Table 1. Conditions and yields of amidoximes 13 and amides 14

\begin{tabular}{cccccc}
\hline $\mathbf{1 , 1 3}, \mathbf{1 4}$ & $\mathrm{R}^{1}$ & $\mathrm{R}^{2}$ & Yield 13 (\%) & Yield 14 (\%) & Reaction time (h) \\
\hline $\mathbf{a}$ & $-\left(\mathrm{CH}_{2}\right)_{3}-$ & 39 & 0 & 7 \\
b & $-\left(\mathrm{CH}_{2}\right)_{5}-$ & 48 & 5 & 80 \\
c & $-o-\mathrm{C}_{6} \mathrm{H}_{4}-\left(\mathrm{CH}_{2}\right)_{2}-$ & 57 & 20 & 80 \\
d & $\mathrm{Ph}$ & $\mathrm{H}$ & 47 & 51 & 100 \\
e & $t-\mathrm{Bu}$ & $\mathrm{H}$ & 0 & 50 & 100 \\
\hline
\end{tabular}

The target amidoximes 13a-d were easily separated from the amides 14 with column chromatography or crystallization. A treatment of the amidoximes 13a-d with aromatic and aliphatic acyl chlorides gave rise to O-acylated intermediates 15 (Scheme 3) one of them was isolated and purified for identification. The other $O$-acylamidoximes 15 were used as crude materials for further cyclization without an additional purification. The cyclization was carried out by action of tetra- $n$-butylammonium fluoride (TBAF) according to literature procedure. ${ }^{35}$ Besides, the product 16f was obtained in reaction of starting amidoxime 13c with excess of trifluoroacetic anhydride as acylating and dehydrating reagent (i.e., without isolation of intermediate 15).

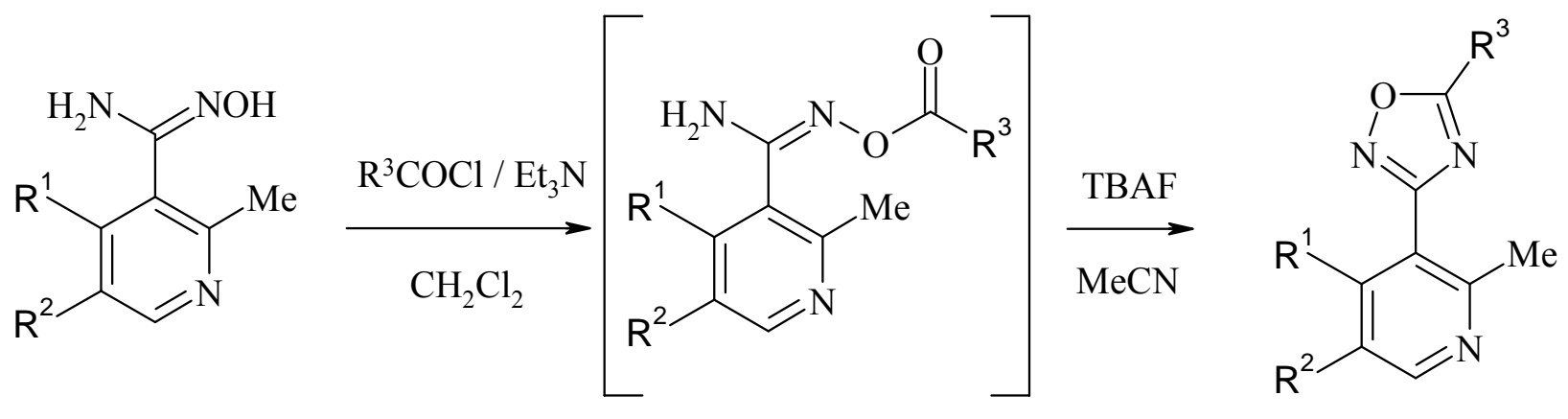

13a-d<smiles>Cc1ncc2c(c1C(N)=NO)-c1ccccc1CC2</smiles>

13c
15

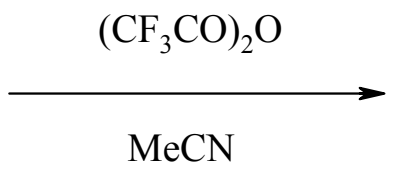
Scheme 3. Synthesis of sterically hindered 3-(1,2,4-oxadiazol-3-yl)pyridines 16.<smiles>Cc1ncc2c(c1-c1noc(C(F)(F)F)n1)-c1ccccc1CC2</smiles>

$16 f$ 
Table 2. Yields of 3-(1,2,4-oxadiazol-3-yl)pyridines 16 (relative to 13)

\begin{tabular}{|c|c|c|c|c|c|}
\hline 13 & 16 & $\mathrm{R}^{1}$ & $\mathrm{R}^{2}$ & $\mathrm{R}^{3}$ & Yield $16(\%)$ \\
\hline $\mathbf{a}$ & $\mathbf{a}$ & \multicolumn{2}{|c|}{$-\left(\mathrm{CH}_{2}\right)_{3}-$} & $\mathrm{Ph}$ & 69 \\
\hline b & b & \multicolumn{2}{|c|}{$-\left(\mathrm{CH}_{2}\right)_{5}-$} & $4-\mathrm{O}_{2} \mathrm{~N}-\mathrm{C}_{6} \mathrm{H}_{4}$ & 78 \\
\hline c & c & \multicolumn{2}{|c|}{$-o-\mathrm{C}_{6} \mathrm{H}_{4}-\left(\mathrm{CH}_{2}\right)_{2}-$} & cyclopropyl & 87 \\
\hline d & d & $\mathrm{Ph}$ & $\mathrm{H}$ & $t-\mathrm{Bu}$ & 81 \\
\hline d & e & $\mathrm{Ph}$ & $\mathrm{H}$ & $\mathrm{CO}_{2} \mathrm{Me}$ & 46 \\
\hline c & f & \multicolumn{2}{|c|}{$-o-\mathrm{C}_{6} \mathrm{H}_{4}-\left(\mathrm{CH}_{2}\right)_{2}-$} & $\mathrm{CF}_{3}$ & 98 \\
\hline
\end{tabular}

The synthesis of sterically hindered 3-(imidazol-2-yl)pyridines 19a,b and 22 utilized the condensation of amidines 17a,b with $\alpha$-haloketones 18, 20 (Schemes 4, 5). However, the conversion of nicotinonitriles $\mathbf{1}$ into amidines $\mathbf{1 7}$ as an initial step of this reaction presented severe difficulty. Most convenient synthesis of amidines from nitriles consist of two steps where a conversion of nitriles into imidates (the Pinner reaction) followed by reaction with amines yields the desired amidines. ${ }^{36}$ Unfortunately, this approach turned out entirely unsuitable for sterically congested nicotinonitriles 1 (vide supra). After several unsuccessful efforts to reveal the conditions for direct reaction of the nicotinonitriles 1 with amines using anhydrous aluminium chloride, ${ }^{37}$ lithium bis(trimethylsilyl)amide ${ }^{38}$ and some other reagents, ${ }^{12,39}$ trimethylaluminum was employed for this reaction. ${ }^{40}$ However, very low yields of the amidines were achieved under the conditions described. ${ }^{40}$ Therefore, we modified these conditions and found that the target amidines 17 can be successfully prepared from the nicotinonitriles $\mathbf{1}$ and amines in the presence of trimethylaluminum using microwave technology (MW) (Scheme 4).

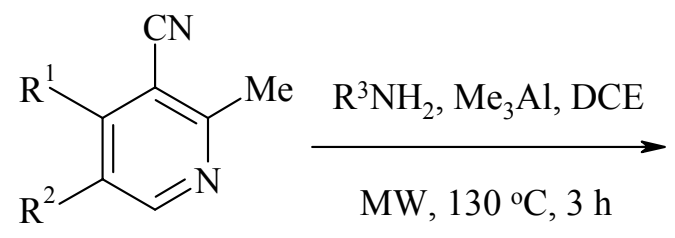

1a,d<smiles>[R]NC(=N)c1c(C)ncc([R])c1[R]</smiles>

17a,b

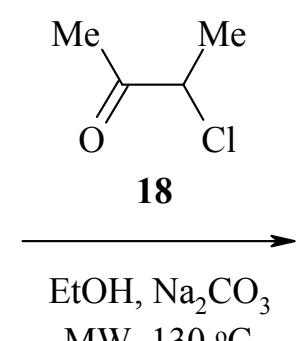

$\mathrm{MW}, 130^{\circ} \mathrm{C}$<smiles>[R]c1cnc(C)c(-c2nc(C)c(C)n2[R])c1[R]</smiles>

19a,b 19a, $\mathrm{R}^{1}=\mathrm{R}^{3}=\mathrm{Ph}, \mathrm{R}^{2}=\mathrm{H}, 6 \mathrm{~h}, 67 \%$

19b, $\mathrm{R}^{1}-\mathrm{R}^{2}=-\left(\mathrm{CH}_{2}\right)_{3}-, \mathrm{R}^{3}=n-\operatorname{Pr}, 4 \mathrm{~h}, 92 \%$

Scheme 4. Synthesis of sterically hindered 3-(imidazol-2-yl)pyridines 19a,b.

The reactions of the amidines $\mathbf{1 7} \mathbf{a}, \mathbf{b}$ with 3-chlorobutan-2-one 18 were also carried out under microwave irradiation yielding directly the desired 3-(imidazol-2-yl)pyridines 19a,b (Scheme 4). At the same time, simple refluxing of mixture of amidine 17a with 3-bromo-1,1,1-trifluoroacetone 20 gave rise to hydroxy derivative $\mathbf{2 1}$ that was easily dehydrated with trifluoroacetic anhydride 
(Scheme 5). Interestingly, the compound 21 existed as a mixture of two stereoisomers. The ${ }^{1} \mathrm{H}$ NMR spectrum of this product 21 was a superposition of two pictures in ratio 57:43 where a noncoincidence of chemical shifts was observed only for the resonances of methyl and methylene groups (see Experimental Section). It is obviously connected with difficulty of rotation at bond "pyridine-imidazoline" since further dehydration gave rise to the sole product $\mathbf{2 2}$. The structure of the final 3-(imidazol-2-yl)pyridine 22 was confirmed by the assignment of ${ }^{1} \mathrm{H}$ and ${ }^{13} \mathrm{C}$ NMR spectra involving ${ }^{1} \mathrm{H}-{ }^{13} \mathrm{C}$ HSQC and HMBC experiments. The position of trifluoromethyl group at position 4 of imidazole ring was established by the cross-peaks in the $2 \mathrm{D}-{ }^{1} \mathrm{H}-{ }^{13} \mathrm{C}-\mathrm{HMBC}$ spectra between 5$\mathrm{H}$ in imidazole nucleus and 1-C in N-phenyl group as well as by interaction NOE in 2D-NOESY spectra between 5-H and ortho-protons of phenyl (Figure 2).<smiles>[M]c1nccc(-c2ccccc2)c1C(=N)Nc1ccccc1</smiles>

17a

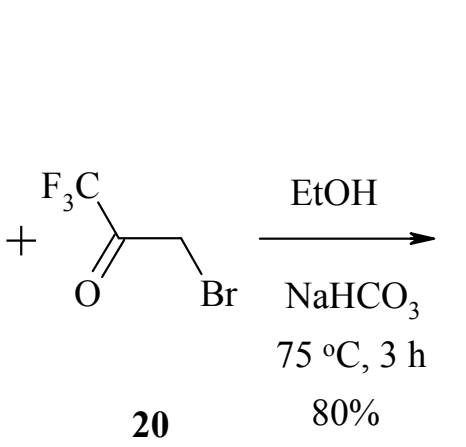

20<smiles>Cc1nccc(-c2ccccc2)c1C1=NC(O)(C(F)(F)F)CN1c1ccccc1</smiles>

21

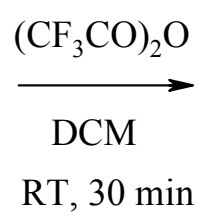

94\%<smiles>Cc1nccc(-c2ccccc2)c1-c1nc(C(F)(F)F)cn1-c1ccccc1</smiles>

22

Scheme 5. Two-step conversion of amidine 17a.

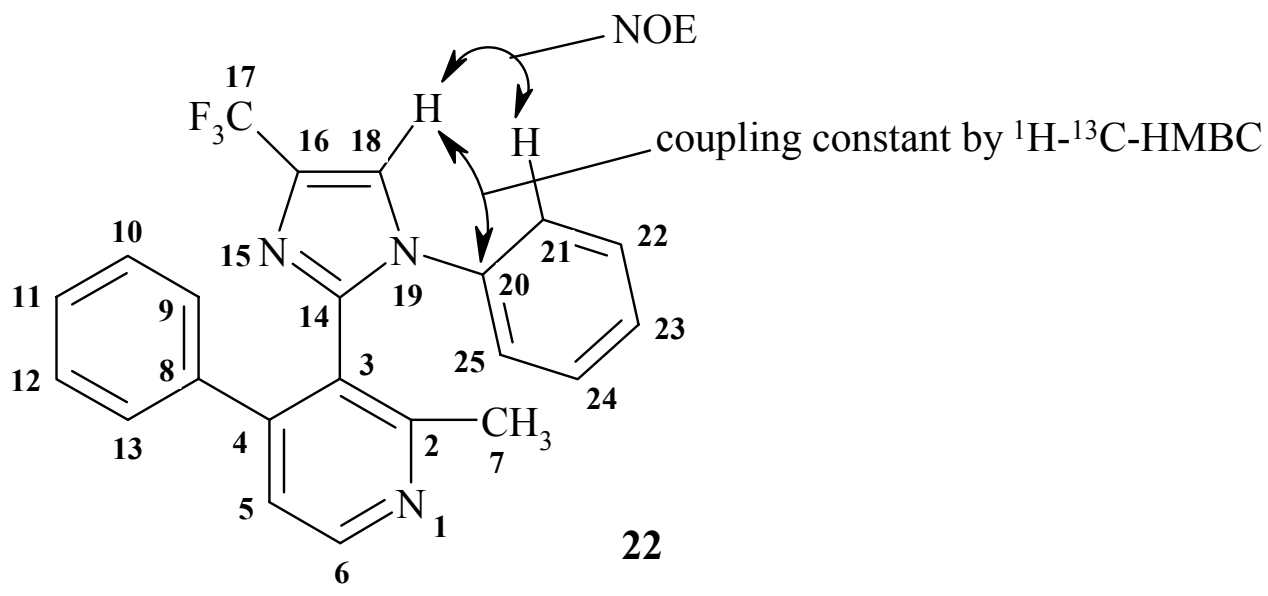

Figure 2. The resonance interactions in structure 22.

Thioamides 23a-i required for synthesis of the sterically hindered 3-(thiazol-2-yl)pyridines 25a$\mathbf{m}$ were prepared by reactions of starting nicotinonitriles 1a-i with $O, O$-diethyldithiophosphoric acid (Scheme 6, Table 3). The latter and similar acids are known as effective reagents for conversion of various nitriles into thioamides in high yields under conditions sufficiently mild to preserve intact a 
wide range of functional groups. ${ }^{41}$ Some conventional methods (e.g., using ammonium sulfide ${ }^{42}$ ) for the transformation of nitriles into thioamides were initially tested but turned out insufficient.<smiles>[R]c1cnc(C)c(C#N)c1C#N</smiles>

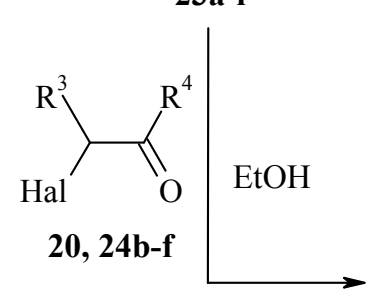<smiles>[R]c1cnc(C)c(-c2nc([R])c([R])s2)c1[R]</smiles><smiles>[R]c1cnc(C)c(C2=NC([R])(O)C([R])S2)c1[R]</smiles>

(CF3CO)2O $\mathrm{CH} 2 \mathrm{Cl} 2$<smiles>Cc1ncc2c(c1C#N)CCC2</smiles>

$1 \mathbf{a}$<smiles>Cc1ncc2c(c1C#N)CCCCC2</smiles>

1b<smiles>Cc1ncc2c(c1C#N)-c1ccccc1CC2</smiles>

1c<smiles>Cc1nccc(-c2ccccc2)c1C#N</smiles>

1d<smiles>Cc1nccc(C(C)(C)C)c1C#N</smiles>

$1 \mathrm{e}$<smiles>Cc1nccc(C(C)C)c1C#N</smiles>

1f<smiles>Cc1ncc2c(c1C#N)CCCC2</smiles>

$1 \mathrm{~g}$<smiles>Cc1ncc2c(c1C#N)C(C)CCC2</smiles>

$1 \mathrm{~h}$<smiles>Cc1ncc2c(c1C#N)-c1ccccc1OC2</smiles>

1i<smiles>CC(=O)C(C)Cl</smiles>

18<smiles>CCOC(=O)C(Cl)C(C)=O</smiles>
20<smiles>O=C(CBr)c1ccc(F)cc1</smiles>

24a<smiles>O=C1CCN(C(=O)c2ccccc2)CC1Br</smiles>

24b<smiles>CCOC(=O)C(=O)CBr</smiles>

$24 c$

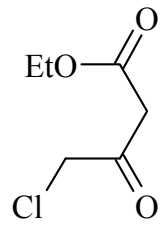

24d<smiles>[M]CC(=O)CBr</smiles>

$24 f$

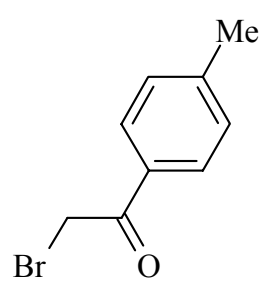

$24 g$

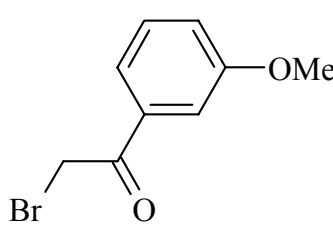

24h

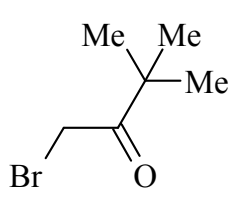

$24 i$

Scheme 6. Synthesis of 3-(thiazol-2-yl)pyridines 25a-m from nicotinonitriles 1a-i and $\alpha-$ haloketones 18, 20, 24a-i. 
Table 3. Conditions and yields of thioamides 23a-i

\begin{tabular}{|c|c|c|c|c|c|}
\hline 1,23 & $\mathrm{R}^{1}$ & $\mathrm{R}^{2}$ & Yield $23(\%)$ & Recovered $1(\%)$ & Reaction time $(\mathrm{h})$ \\
\hline $\mathbf{a}$ & \multicolumn{2}{|c|}{$-\left(\mathrm{CH}_{2}\right)_{3}-$} & 65 & 21 & 8 \\
\hline b & \multicolumn{2}{|c|}{$-\left(\mathrm{CH}_{2}\right)_{5}-$} & 45 & 17 & 20 \\
\hline c & \multicolumn{2}{|c|}{$-o-\mathrm{C}_{6} \mathrm{H}_{4}-\left(\mathrm{CH}_{2}\right)_{2}-$} & 62 & - & 100 \\
\hline d & $\mathrm{Ph}$ & $\mathrm{H}$ & 85 & 10 & 8 \\
\hline e & $t-\mathrm{Bu}$ & $\mathrm{H}$ & 39 & 59 & 100 \\
\hline $\mathbf{f}$ & $i-\operatorname{Pr}$ & $\mathrm{H}$ & 68 & 30 & 40 \\
\hline $\mathbf{g}$ & \multicolumn{2}{|c|}{$-\left(\mathrm{CH}_{2}\right)_{4}-$} & 60 & 25 & 20 \\
\hline $\mathbf{h}$ & \multicolumn{2}{|c|}{$-\mathrm{CH}\left(\mathrm{CH}_{3}\right)\left(\mathrm{CH}_{2}\right)_{3}-$} & 66 & 18 & 14 \\
\hline $\mathbf{i}$ & \multicolumn{2}{|c|}{$-o-\mathrm{C}_{6} \mathrm{H}_{4}-\mathrm{OCH}_{2}-$} & 44 & - & 40 \\
\hline
\end{tabular}

It should be noted that the complete conversion of nitriles 1a-i into amidines 23a-i was not achieved in all the experiments even by long-term heating of reaction mixtures (up to $100 \mathrm{~h}$ ). Some amount of a starting nitrile 1 remained and can be recovered in many cases (Table 3 ). Moreover, the conversion decreased if the temperature of reaction mixture was elevated to $100-120{ }^{\circ} \mathrm{C}$. It can be explained by thermal decomposition of thioamides 23 to starting nitriles $\mathbf{1}$ and $\mathrm{H}_{2} \mathrm{~S} .{ }^{43}$ It is known also that a heating of thioamides with pyridine caused an analogous decomposition; ${ }^{44}$ it is not improbable that a similar effect can have the starting cyanopyridines $\mathbf{1}$. Unfortunately, our attempts to increase the conversion and improve the yields of the desired thioamides 23a-i using microwave technology turned out unsuccessful.

Table 4. Yields of 3-(thiazol-2-)pyridines 25a-m

\begin{tabular}{|c|c|c|c|c|c|c|}
\hline $\begin{array}{l}\text { Thio- } \\
\text { amide }\end{array}$ & $\begin{array}{c}\alpha \text {-Halo- } \\
\text { ketone }\end{array}$ & $\mathrm{R}^{1}$ & $\mathrm{R}^{3}$ & $\mathrm{R}^{4}$ & $\begin{array}{c}\text { Yield } 26 \\
(\%)\end{array}$ & $\begin{array}{c}\text { Yield } 25 \\
(\%)\end{array}$ \\
\hline $23 a$ & $24 a$ & $-\left(\mathrm{CH}_{2}\right)_{3}-$ & $\mathrm{H}$ & $4-\mathrm{F}-\mathrm{C}_{6} \mathrm{H}_{4}$ & - & 86 (25a) \\
\hline $23 a$ & $24 b$ & $-\left(\mathrm{CH}_{2}\right)_{3}-$ & $-\left(\mathrm{CH}_{2}\right)$ & $(\mathrm{Boc})\left(\mathrm{CH}_{2}\right)_{2}-$ & 64 (26a) & $52^{\mathrm{a}}(\mathbf{2 5 b})$ \\
\hline $23 b$ & $24 a$ & $-\left(\mathrm{CH}_{2}\right)_{5}-$ & $\mathrm{H}$ & $4-\mathrm{F}-\mathrm{C}_{6} \mathrm{H}_{4}$ & - & $99(25 c)$ \\
\hline $23 c$ & 18 & $-o-\mathrm{C}_{6} \mathrm{H}_{4}-\left(\mathrm{CH}_{2}\right)_{2}-$ & $\mathrm{Me}$ & $\mathrm{Me}$ & - & $82(\mathbf{2 5 d})$ \\
\hline 23d & 20 & $\begin{array}{ll}\mathrm{Ph} & \mathrm{H}\end{array}$ & $\mathrm{H}$ & $\mathrm{CF}_{3}$ & $90(\mathbf{2 6 b})$ & $71^{\mathrm{a}}(\mathbf{2 5 e})$ \\
\hline 23d & $24 c$ & $\mathrm{Ph}$ & $\mathrm{H}$ & $\mathrm{CO}_{2} \mathrm{Et}$ & $73(\mathbf{2 6 c})$ & $83^{\mathrm{a}}(\mathbf{2 5 f})$ \\
\hline 23d & 24d & $\mathrm{Ph}$ & $\mathrm{H}$ & $\mathrm{CH}_{2} \mathrm{CO}_{2} \mathrm{Et}$ & $100(\mathbf{2 6 d})$ & $88^{\mathrm{a}}(\mathbf{2 5 g})$ \\
\hline 23d & $24 \mathrm{e}$ & $\mathrm{Ph}$ & $\mathrm{CO}_{2} \mathrm{Et}$ & $\mathrm{CH}_{3}$ & $72(\mathbf{2 6 e})$ & $68^{\mathrm{a}}(\mathbf{2 5 h})$ \\
\hline $23 e$ & $24 f$ & $t-\mathrm{Bu}$ & $\mathrm{H}$ & Et & $90(\mathbf{2 6 f})$ & $71^{\mathrm{a}}(\mathbf{2 5 i})$ \\
\hline $23 f$ & $24 g$ & $i-\operatorname{Pr}$ & $\mathrm{H}$ & 4- $\mathrm{CH}_{3} \mathrm{C}_{6} \mathrm{H}_{4}$ & - & $90(\mathbf{2 5} \mathbf{j})$ \\
\hline $23 g$ & $24 \mathrm{~g}$ & $-\left(\mathrm{CH}_{2}\right)_{4}-$ & $\mathrm{H}$ & $4-\mathrm{CH}_{3} \mathrm{C}_{6} \mathrm{H}_{4}$ & - & 80 (25k) \\
\hline $23 h$ & $24 h$ & $-\mathrm{CH}\left(\mathrm{CH}_{3}\right)\left(\mathrm{CH}_{2}\right)_{3}-$ & $\mathrm{H}$ & $3-\mathrm{CH}_{3} \mathrm{OC}_{6} \mathrm{H}_{4}$ & - & 98 (25l) \\
\hline $23 \mathbf{i}$ & $24 \mathbf{i}$ & $-o-\mathrm{C}_{6} \mathrm{H}_{4}-\mathrm{OCH}_{2}-$ & $\mathrm{H}$ & $t-\mathrm{Bu}$ & - & $46(\mathbf{2 5 m})$ \\
\hline
\end{tabular}

${ }^{a}$ Yields from the starting thioamides 23. 
The series of sterically hindered 3-(thiazol-2-yl)pyridines $\mathbf{2 5 a - m}$ was synthesized by wellknown reactions ${ }^{45}$ of thioamides 23a-i with $\alpha$-haloketones 18, 20, 24a-i (Scheme 8). The reactions were carried out in anhydrous ethanol to form hydrohalogenides of the target 3-(thiazol-2yl)pyridines 25a-m (Table 4). At the same time, intermediate alcohols 26a-f were isolated in a number of cases (Scheme 8, Table 4). These alcohols 26a-f were easily dehydrated by trifluoroacetic anhydride according to known procedures. ${ }^{46}$

\section{Conclusions}

In summary, we have developed procedures to convert a series of readily available sterically hindered nicotinonitriles into the corresponding 2,4-disubstituted 3-(1,2,4-oxadiazol-3-yl)-, 3(thiazol-2-yl)- and 3-(imidazol-2-yl)pyridines. These compounds together with previously described 3-(5-tetrazolyl)pyridines ${ }^{27}$ and 3-(1,3,4-oxadiazol-2-yl)pyridines ${ }^{27}$ could constitute a basis for a potential combinatorial library. This entire library containing more than 200 compounds was tested by the computer software PASS (Prediction of Activity Spectra for Substances). ${ }^{47}$ This program illustrates the predicted activity spectrum of a compound as probability of activity $\left(P_{a}\right)$ and probability of inactivity $\left(P_{i}\right)$. For example, it was predicted by the PASS that 3-(1,2,4-oxadiazol-3yl)pyridines 16 can possess an antidepressant property, and anxiolytic activity for five of the tested six examples with $P_{a}$ more than $70 \%$. Our further investigations will be directed to the synthesis of the compounds with the potential activity predicted, and then to a pharmacological evaluation of the latter.

\section{Experimental Section}

General Procedures. Starting nicotinonitriles 1a-i were prepared according to literature procedure. ${ }^{48}$ All the acid chlorides, $\alpha$-haloketones and other reagents are commercially available. The reactions were monitored by TLC (aluminium sheets, silica gel $60 \mathrm{~F}_{254}$, Merck). Merck Kieselgel 60 (230-400 mesh) was used for a column chromatography. ${ }^{1} \mathrm{H}$ NMR spectra were recorded on a Bruker Avance DRX $400(400.13 \mathrm{MHz})$ spectrometer equipped with a 5 mm inverse multinuclear gradient probehead in DMSO- $d_{6}$ or $\mathrm{CDCl}_{3}$, and the chemical shifts $(\delta)$ are given in ppm relative to the signal for TMS as internal standard. ${ }^{13} \mathrm{C}$ NMR spectrum was recorded on the same instrument at $100.61 \mathrm{MHz}$ using DMSO- $d_{6}$ as solvent. The assignments of signals in ${ }^{1} \mathrm{H}$ and ${ }^{13} \mathrm{C}$ NMR spectra were performed using HSQC, HMBC and NOESY experiments. Mass spectra were recorded in the course of LCMS measurements and were obtained by atmosphere pressure chemical ionization (APCI method, positive mode) on an 1100 LCMSD (Agilent Technologies) instrument. IR spectra were measured on an EQUINOX 55 Bruker spectrometer. Elemental analyses were carried out, using CARLO-ERBA 1106 and 1500 automatic elemental analyzers, by the Analytical Laboratory in the A.N. Nesmeyanov Institute of Organoelement Compounds (INEOS RAS, Vavilova str. 28, 119991, Moscow, Russia). Melting points were determined by open glass capillary method and were uncorrected. Milestone Ethos SYNTH (reactors MPR 600/12S and PRO- 
24) and CEM Discover microwave labstations (both operating at $2450 \mathrm{MHz}$ under continuous internal temperature control) were used for the experimental and scale-up reactions.

$\mathrm{N}$-Hydroxy-3-methyl-6,7-dihydro-5H-cyclopenta[c]pyridine-4-carboximidamide (13a). Aqueous $50 \%$ solution of hydroxylamine $(1.0 \mathrm{~mL}, 17 \mathrm{mmol})$ was added under stirring to the solution of 3methyl-6,7-dihydro-5H-cyclopenta[c]pyridine-4-carbonitrile $1 \mathrm{a}(0.6 \mathrm{~g}, 3.8 \mathrm{mmol})$ in ethanol (20 $\mathrm{mL})$. The mixture obtained was stirred at $75{ }^{\circ} \mathrm{C}$ for $7 \mathrm{~h}$ and then concentrated under reduced pressure. The residue was diluted with ethyl acetate $(35 \mathrm{~mL})$. Precipitate formed was filtered off, dried and recrystallized from ethanol to give the titled product 13a $(0.28 \mathrm{~g}, 39 \%)$ as white powder; mp 114-116 ${ }^{\circ} \mathrm{C} ;{ }^{1} \mathrm{H}$ NMR (400 MHz, DMSO- $\left.d_{6}\right) \delta$ 1.94-2.07 (m, 2H, $\mathrm{CH}_{2}$ ), 2.42 (s, 3H, Me), 2.78$2.93\left(\mathrm{~m}, 4 \mathrm{H}, 2 \mathrm{CH}_{2}\right), 5.78\left(\right.$ br s, $\left.2 \mathrm{H}, \mathrm{NH}_{2}\right), 8.28(\mathrm{~s}, 1 \mathrm{H}, 6-\mathrm{H}), 9.27(\mathrm{~s}, 1 \mathrm{H}, \mathrm{OH})$. IR (film) $v 3506$ $(\mathrm{OH}), 3290(\mathrm{NH}), 3168(\mathrm{OH}), 2916(\mathrm{CH}), 1620(\mathrm{C}=\mathrm{N}){ }^{49} \mathrm{~cm}^{-1}$. APCI MS m/z: $192[\mathrm{M}+\mathrm{H}]^{+}$. Anal. Calcd for $\mathrm{C}_{10} \mathrm{H}_{13} \mathrm{~N}_{3} \mathrm{O}$ (191.23): C, 62.81; H, 6.85; N, 21.97\%. Found: C, 62.62; H, 7.01; N, 22.02\%. $\mathrm{N}$-Hydroxy-3-methyl-6,7,8,9-tetrahydro-5H-cyclohepta[c]pyridine-4-carboximidamide (13b). Aqueous $50 \%$ solution of hydroxylamine $(2.84 \mathrm{~mL}, 48.2 \mathrm{mmol})$ was added under stirring to the solution of 3-methyl-6,7,8,9-tetrahydro-5H-cyclohepta[c]pyridine-4-carbonitrile $\mathbf{1 b}(2.0 \mathrm{~g}, 10.7$ $\mathrm{mmol})$ in ethanol $(40 \mathrm{~mL})$. The mixture was refluxed under stirring for $80 \mathrm{~h}$ and then evaporated to dryness. The residue was diluted with ethyl acetate $(70 \mathrm{~mL})$. Precipitate formed was filtered off, dried and recrystallized from ethanol to give the product $\mathbf{1 3 b}(1.13 \mathrm{~g}, 48 \%)$ as white powder; mp 254-255 ${ }^{\circ} \mathrm{C}$ (decomp.); ${ }^{1} \mathrm{H}$ NMR (400 MHz, DMSO- $\left.d_{6}\right) \delta 1.44-1.64$ (m, 4H, $\mathrm{CH}_{2} \mathrm{CH}_{2}$ ), 1.73-1.86 (m, 2H, $\left.\mathrm{CH}_{2}\right), 2.38$ (s, 3H, Me), 2.66-2.78 (m, 4H, $\left.\mathrm{CH}_{2} \mathrm{CH}_{2}\right), 5.83\left(\mathrm{~s}, 2 \mathrm{H}, \mathrm{NH}_{2}\right), 8.15(\mathrm{~s}, 1 \mathrm{H}, 6-\mathrm{H})$, $9.23(\mathrm{~s}, 1 \mathrm{H}, \mathrm{OH})$. IR (film) v $3461(\mathrm{OH}), 3279(\mathrm{NH}), 3151(\mathrm{OH}), 2912(\mathrm{CH}), 1653(\mathrm{C}=\mathrm{N}) \mathrm{cm}^{-1}$. APCI MS $m / z: 220[\mathrm{M}+\mathrm{H}]^{+}$. Anal. Calcd for $\mathrm{C}_{12} \mathrm{H}_{17} \mathrm{~N}_{3} \mathrm{O}$ (219.29): C, 65.73; H, 7.81; N, 19.16\%. Found: C, 65.55; H, 7.68; N, 19.05\%.

The filtrate was concentrated under reduced pressure, and the residue was chromatographed (silica gel, ethyl acetate) to afford the starting nitrile $\mathbf{1 b}(0.3 \mathrm{~g}, 15 \%)$, amidoxime $\mathbf{1 3 b}(0.12 \mathrm{~g}, 5 \%)$ and 3methyl-6,7,8,9-tetrahydro-5H-cyclohepta[c]pyridine-4-carboxamide (14b). $(0.11 \mathrm{~g}, 5 \%)$ as white solid; mp 172-173 ${ }^{\circ} \mathrm{C}$ (from ethanol); ${ }^{1} \mathrm{H}$ NMR (400 MHz, DMSO- $\left.d_{6}\right) \delta 1.47-1.61$ (m, 4H, $\mathrm{CH}_{2} \mathrm{CH}_{2}$ ), 1.73-1.84 (m, 2H, $\left.\mathrm{CH}_{2}\right), 2.38(\mathrm{~s}, 3 \mathrm{H}, \mathrm{Me}), 2.65-2.78\left(\mathrm{~m}, 4 \mathrm{H}, \mathrm{CH}_{2} \mathrm{CH}_{2}\right), 7.56$ and 9.23 (both br. s, $1 \mathrm{H}$ and $\left.1 \mathrm{H}, \mathrm{NH}_{2}\right), 8.14(\mathrm{~s}, 1 \mathrm{H}, 6-\mathrm{H})$. IR (film) v $3451(\mathrm{NH}), 2908(\mathrm{CH}), 1642(\mathrm{C}=\mathrm{O}) \mathrm{cm}^{-}$ 1. APCI MS $m / z: 205[\mathrm{M}+\mathrm{H}]^{+}$. Anal. Calcd for $\mathrm{C}_{12} \mathrm{H}_{16} \mathrm{~N}_{2} \mathrm{O}$ (204.27): C, 70.56; H, 7.90; N, 13.71\%. Found: C, 70.33; H, 8.00; N, 13.80\%.

$\mathrm{N}$-Hydroxy-2-methyl-5,6-dihydrobenzo[f]isoquinoline-1-carboximidamide (13c). Aqueous $50 \%$ solution of hydroxylamine $(2.4 \mathrm{~mL}, 41 \mathrm{mmol})$ was added under stirring to the solution of 2-methyl5,6-dihydrobenzo[f] isoquinoline-1-carbonitrile 1c $(2.0 \mathrm{~g}, 9.1 \mathrm{mmol})$ in ethanol $(40 \mathrm{~mL})$. The mixture obtained was refluxed under stirring for $80 \mathrm{~h}$ and then evaporated to dryness. The residue was chromatographed (silica gel, chloroform/methanol 95:5) to give the product 13c $(1.31 \mathrm{~g}, 57 \%)$ as pale-yellow solid; mp 256-257 ${ }^{\circ} \mathrm{C}$ (from ethanol); ${ }^{1} \mathrm{H}$ NMR (400 MHz, DMSO- $\left.d_{6}\right) \delta 2.49(\mathrm{~s}, 3 \mathrm{H}$, $\mathrm{Me})$, 2.64-2.85 (m, 4H, $\left.\mathrm{CH}_{2} \mathrm{CH}_{2}\right), 6.03\left(\mathrm{~s}, 2 \mathrm{H}, \mathrm{NH}_{2}\right), 7.19-7.37\left(\mathrm{~m}, 3 \mathrm{H}, \mathrm{C}_{6} \mathrm{H}_{4}\right), 8.14(\mathrm{~d}, 1 \mathrm{H}, J 7.8$ $\left.\mathrm{Hz}, \mathrm{C}_{6} \mathrm{H}_{4}\right), 8.38(\mathrm{~s}, 1 \mathrm{H}, 6-\mathrm{H}), 9.28(\mathrm{~s}, 1 \mathrm{H}, \mathrm{OH})$. IR (film) v $3489(\mathrm{OH}), 3386(\mathrm{NH}), 3179(\mathrm{OH})$, $2938(\mathrm{CH}), 1653(\mathrm{C}=\mathrm{N}) \mathrm{cm}^{-1}$. APCI MS m/z: $254[\mathrm{M}+\mathrm{H}]^{+}$. Anal. Calcd for $\mathrm{C}_{15} \mathrm{H}_{15} \mathrm{~N}_{3} \mathrm{O}(253.31)$ : , 
71.13; H, 5.97; N, 16.59\%. Found: C, 71.11; H, 5.93; N, 16.42\%. Another product isolated from chromatography column was identified as 2-methyl-5,6-dihydrobenzo[f]isoquinoline-1carboxamide (14c) (0.44 g, 20\%), white powder; mp 203-205 ${ }^{\circ} \mathrm{C}$ (from ethanol); ${ }^{1} \mathrm{H}$ NMR (400 $\left.\mathrm{MHz}, \mathrm{DMSO}-d_{6}\right) \delta 2.49(\mathrm{~s}, 3 \mathrm{H}, \mathrm{Me}), 2.65-2.85\left(\mathrm{~m}, 4 \mathrm{H}, \mathrm{CH}_{2} \mathrm{CH}_{2}\right), 7.25-7.37\left(\mathrm{~m}, 3 \mathrm{H}, \mathrm{C}_{6} \mathrm{H}_{4}\right), 7.67$ and 7.93 (both br. s, $1 \mathrm{H}$ and $\left.1 \mathrm{H}, \mathrm{NH}_{2}\right), 8.05\left(\mathrm{~d}, 1 \mathrm{H}, J 7.8 \mathrm{~Hz}, \mathrm{C}_{6} \mathrm{H}_{4}\right), 8.37$ (s, 1H, 6-H). IR (film) $v$ $3366(\mathrm{NH}), 2947(\mathrm{CH}), 1659(\mathrm{C}=\mathrm{O}) \mathrm{cm}^{-1}$. APCI MS m/z: $239[\mathrm{M}+\mathrm{H}]^{+}$. Anal. Calcd for $\mathrm{C}_{15} \mathrm{H}_{14} \mathrm{~N}_{2} \mathrm{O}$ (238.29): C, 75.61; H, 5.92; N, 11.76\%. Found: C, 75.51; H, 5.76; N, 11.65\%.

$\mathrm{N}$-Hydroxy-2-methyl-4-phenylpyridine-3-carboximidamide (13d). Aqueous $50 \%$ solution of hydroxylamine $(2.7 \mathrm{~mL}, 46 \mathrm{mmol})$ was added under stirring to the solution of 2-methyl-4phenylnicotinonitrile $1 \mathrm{~d}(2.0 \mathrm{~g}, 10.3 \mathrm{mmol})$ in ethanol $(40 \mathrm{~mL})$. The mixture obtained was refluxed under stirring for $100 \mathrm{~h}$ and then evaporated to dryness. The residue was chromatographed (silica gel, chloroform/methanol 94:6) to give the product 13d (1.10 g, 47\%) as pale-yellow powder; mp 206-207 ${ }^{\circ} \mathrm{C}$ (from ethanol); ${ }^{1} \mathrm{H}$ NMR (400 MHz, DMSO- $\left.d_{6}\right) \delta 2.53$ (s, 3H, Me), $5.90\left(\mathrm{~s}, 2 \mathrm{H}, \mathrm{NH}_{2}\right.$ ), $7.21(\mathrm{~d}, 1 \mathrm{H}, J 5.0 \mathrm{~Hz}, 5-\mathrm{H}), 7.34-7.44$ (m, 3H, Ph), 7.52-7.57 (m, 2H, Ph), 8.48 (d, 1H, J 5.0 Hz, 6$\mathrm{H}), 9.17(\mathrm{~s}, 1 \mathrm{H}, \mathrm{OH})$. IR (film) v $3451(\mathrm{OH}), 3297(\mathrm{NH}), 3178(\mathrm{OH}), 1666(\mathrm{C}=\mathrm{N}) \mathrm{cm}^{-1}$. APCI MS $m / z: 228[\mathrm{M}+\mathrm{H}]^{+}$. Anal. Calcd for $\mathrm{C}_{13} \mathrm{H}_{13} \mathrm{~N}_{3} \mathrm{O}$ (227.27): C, 68.71; H, 5.77; N, 18.49\%. Found: C, $68.56 ; \mathrm{H}, 5.61 ; \mathrm{N}, 18.35 \%$. Another product isolated from chromatography column was identified as 2-methyl-4-phenylnicotinamide (14d) (1.12 g, 51\%), white powder; mp 174-176 ${ }^{\circ} \mathrm{C}$ (from ethanol); ${ }^{1} \mathrm{H}$ NMR (400 MHz, DMSO- $\left.d_{6}\right) \delta 2.53$ (s, 3H, Me), 7.22 (d, $\left.1 \mathrm{H}, J 5.1 \mathrm{~Hz}, 5-\mathrm{H}\right), 7.37-7.57$ $(\mathrm{m}, 5 \mathrm{H}, \mathrm{Ph}), 7.49$ and 7.82 (both $\mathrm{s}, 1 \mathrm{H}$ and $\left.1 \mathrm{H}, \mathrm{NH}_{2}\right), 8.48(\mathrm{~d}, 1 \mathrm{H}, J 5.1 \mathrm{~Hz}, 6-\mathrm{H})$. IR (film) $v 3306$ $(\mathrm{NH}), 1698(\mathrm{C}=\mathrm{O}) \mathrm{cm}^{-1}$. APCI MS m/z: $213[\mathrm{M}+\mathrm{H}]^{+}$. Anal. Calcd for $\mathrm{C}_{13} \mathrm{H}_{12} \mathrm{~N}_{2} \mathrm{O}(212.25)$ : C, 73.57; H, 5.70; N, 13.20\%. Found: C, 73.59; H, 5.73; N, 13.28\%.

4-tert-Butyl-2-methylnicotinamide (14e). Aqueous 50\% solution of hydroxylamine $(2.36 \mathrm{~mL}, 40$ mmol) was added under stirring to the solution of 4-tert-butyl-2-methylnicotinonitrile $1 \mathrm{e}(2.0 \mathrm{~g}$, $11.5 \mathrm{mmol})$ in ethanol $(40 \mathrm{~mL})$. The mixture obtained was refluxed under stirring for $100 \mathrm{~h}$ and then evaporated to dryness. The residue was chromatographed (silica gel, ethyl acetate) to give the starting nitrile $1 \mathrm{e}(0.9 \mathrm{~g}, 45 \%)$ and the titled product $14 \mathrm{e}(1.10 \mathrm{~g}, 50 \%$ in regard to the initial amount of 1e, or $91 \%$ taking into account the conversion level) as white powder; mp $164-165{ }^{\circ} \mathrm{C}$ (from EtOAc); ${ }^{1} \mathrm{H}$ NMR (400 MHz, DMSO- $\left.d_{6}\right) \delta 1.37(\mathrm{~s}, 9 \mathrm{H}, t-\mathrm{Bu}), 2.45$ (s, 3H, Me), $7.24(\mathrm{~d}, 1 \mathrm{H}, J 5.4$ $\mathrm{Hz}, 5-\mathrm{H}$ ), 7.62 and 7.92 (both br. s, $1 \mathrm{H}$ and $1 \mathrm{H}, \mathrm{NH}_{2}$ ), 8.31 (d, 1H, J 5.4 Hz, 6-H). IR (film) $v 3471$ $(\mathrm{NH}), 2970(\mathrm{CH}), 1686(\mathrm{C}=\mathrm{O}) \mathrm{cm}^{-1}$. APCI MS m/z: $193[\mathrm{M}+\mathrm{H}]^{+}$. Anal. Calcd for $\mathrm{C}_{11} \mathrm{H}_{16} \mathrm{~N}_{2} \mathrm{O}$ (192.26): C, 68.72; H, 8.39; N, 14.57\%. Found: C, 68.51; H, 8.29; N, 14.68\%.

3-Methyl-4-(5-phenyl-1,2,4-oxadiazol-3-yl)-6,7-dihydro-5H-cyclopenta[c]pyridine

(16a). Benzoyl chloride $(0.25 \mathrm{~g}, 0.21 \mathrm{~mL}, 1.8 \mathrm{mmol})$ was added dropwise at $0{ }^{\circ} \mathrm{C}$ to cooled solution of $N$ hydroxy-3-methyl-6,7-dihydro-5H-cyclopenta[c]pyridine-4-carboximidamide 13a (0.28 g, 1.46 mmol) and triethylamine $(0.3 \mathrm{~g}, 0.42 \mathrm{~mL}, 3.0 \mathrm{mmol})$ in anhydrous dichloromethane $(4 \mathrm{~mL})$ under stirring. The reaction mixture was then stirred at room temperature for $14 \mathrm{~h}$ and poured into saturated aqueous solution of $\mathrm{NaHCO}_{3}(50 \mathrm{~mL})$. The product was extracted with ethyl acetate $(6 \times 5$ $\mathrm{mL}$ ), and the extract was dried over $\mathrm{MgSO}_{4}$ and concentrated under reduced pressure. The residue was chromatographed (silica gel, chloroform/methanol 9:1) to furnish the intermediate $15[N-$ (benzoyloxy)-3-methyl-6,7-dihydro-5H-cyclopenta[c]pyridine-4-carboximidamide] $\left(\mathrm{R}^{3}=\mathrm{Ph}\right)$ 
$(0.38 \mathrm{~g}, 88 \%)$ as pale-brown crystal solid; $\mathrm{mp} 185-186{ }^{\circ} \mathrm{C} ;{ }^{1} \mathrm{H}$ NMR (400 MHz, DMSO- $\left.d_{6}\right) \delta 1.99-$ $2.10\left(\mathrm{~m}, 2 \mathrm{H}, \mathrm{CH}_{2}\right), 2.51$ (s, 3H, Me), 2.88-2.97 (m, 4H, 2CH $), 7.00\left(\mathrm{~s}, 2 \mathrm{H}, \mathrm{NH}_{2}\right), 7.50-7.57(\mathrm{~m}, 2 \mathrm{H}$, $\mathrm{Ph})$, 7.63-7.69 (m, 1H, Ph), 8.15-8.20 (m, 2H, Ph), 8.38 (s, 1H, 6-H).

The intermediate 15 was then suspended in anhydrous acetonitrile $(2 \mathrm{~mL})$, and tetra- $n$ butylammonium fluoride trihydrate (TBAF) $(0.46 \mathrm{~g}, 1.46 \mathrm{mmol})$ was added to the suspension. The reaction mixture was stirred at room temperature for $12 \mathrm{~h}$ then concentrated under reduced pressure. The residue was chromatographed (silica gel, hexane/ethyl acetate in gradient 30 to 50\%) to give the product 16a $\left(0.28 \mathrm{~g}, 69 \%\right.$ in regard to the starting 13a) as light gray crystal solid; mp $75-76{ }^{\circ} \mathrm{C}$; ${ }^{1} \mathrm{H}$ NMR (400 MHz, DMSO-d $)_{6} \delta$ 2.01-2.11 (m, 2H, $\mathrm{CH}_{2}$ ), 2.63 (s, 3H, Me), 2.97 (t, 2H, J 7.5 Hz, $\mathrm{CH}_{2}$ ), 3.05 (t, 2H, J 7.5 Hz, $\mathrm{CH}_{2}$ ), 7.64-7.70 (m, 2H, Ph), 7.71-7.78 (m, 1H, Ph), 8.16-8.21 (m, 2H, $\mathrm{Ph}), 8.49(\mathrm{~s}, 1 \mathrm{H}, 6-\mathrm{H})$. APCI MS $m / z: 278[\mathrm{M}+\mathrm{H}]^{+}$. Anal. Calcd for $\mathrm{C}_{17} \mathrm{H}_{15} \mathrm{~N}_{3} \mathrm{O}$ (277.33): C, 73.63; H, 5.45; N, 15.15\%. Found: C, 73.47; H, 5.49; N, 15.04\%.

\section{3-Methyl-4-[5-(4-nitrophenyl)-1,2,4-oxadiazol-3-yl]-6,7,8,9-tetrahydro-5H-cyclohepta[c]pyridine}

(16b). 4-Nitrobenzoyl chloride $(0.186 \mathrm{~g}, 1.0 \mathrm{mmol})$ was added to solution of the precursor $\mathbf{1 3 b}(0.20$ $\mathrm{g}, 0.91 \mathrm{mmol})$ and triethylamine $(0.15 \mathrm{~g}, 0.21 \mathrm{~mL}, 1.5 \mathrm{mmol})$ in anhydrous dichloromethane $(2 \mathrm{~mL})$ under stirring. The reaction mixture was stirred at room temperature for $10 \mathrm{~h}$ then poured into saturated aqueous solution of $\mathrm{NaHCO}_{3}(10 \mathrm{~mL})$. The product was extracted with ethyl acetate $(7 \times 5$ $\mathrm{mL}$ ), and the extract was dried over $\mathrm{MgSO}_{4}$ and concentrated under reduced pressure. The residue was suspended in anhydrous acetonitrile $(2 \mathrm{~mL})$, and TBAF $(0.32 \mathrm{~g}, 1.00 \mathrm{mmol})$ was added to the suspension. The reaction mixture was stirred at room temperature for $3 \mathrm{~h}$ then concentrated under reduced pressure. The residue was chromatographed (silica gel, hexane/ethyl acetate 1:1) to give the product 16b (0.25 g, 78\%) as pale-yellow crystal solid; mp 104-106 ${ }^{\circ} \mathrm{C} ;{ }^{1} \mathrm{H}$ NMR (400 $\mathrm{MHz}$, DMSO-d $\left.d_{6}\right) \delta 1.48-1.65\left(\mathrm{~m}, 4 \mathrm{H}, \mathrm{CH}_{2} \mathrm{CH}_{2}\right), 1.75-1.84\left(\mathrm{~m}, 2 \mathrm{H}, \mathrm{CH}_{2}\right), 2.33(\mathrm{~s}, 3 \mathrm{H}, \mathrm{Me}), 2.59-2.65(\mathrm{~m}$, $\left.2 \mathrm{H}, \mathrm{CH}_{2}\right), 2.82-2.89\left(\mathrm{~m}, 2 \mathrm{H}, \mathrm{CH}_{2}\right), 8.38(\mathrm{~s}, 1 \mathrm{H}, 6-\mathrm{H}), 8.41-8.50\left(\mathrm{~m}, 4 \mathrm{H}, \mathrm{C}_{6} \mathrm{H}_{4}\right)$. APCI MS $m / z: 351$ $[\mathrm{M}+\mathrm{H}]^{+}$. Anal. Calcd for $\mathrm{C}_{19} \mathrm{H}_{18} \mathrm{~N}_{4} \mathrm{O}_{3}$ (350.38): C, 65.13; H, 5.18; N, 15.99\%. Found: C, 65.11; H, $5.23 ; \mathrm{N}, 15.89 \%$.

1-(5-Cyclopropyl-1,2,4-oxadiazol-3-yl)-2-methyl-5,6-dihydrobenzo[f]isoquinoline (16c). Cyclopropanecarbonyl chloride $(0.125 \mathrm{~g}, 1.2 \mathrm{mmol})$ was added at $0{ }^{\circ} \mathrm{C}$ to solution of the intermediate $13 \mathrm{c}(0.25 \mathrm{~g}, 1.0 \mathrm{mmol})$ and triethylamine $(0.15 \mathrm{~g}, 0.21 \mathrm{~mL}, 1.5 \mathrm{mmol})$ in anhydrous dichloromethane $(2 \mathrm{~mL})$ under stirring. The reaction mixture was stirred at room temperature for 10 $\mathrm{h}$ then poured into saturated aqueous solution of $\mathrm{NaHCO}_{3}(10 \mathrm{~mL})$. The product was extracted with ethyl acetate $(4 \times 5 \mathrm{~mL})$, and the extract was dried over $\mathrm{MgSO}_{4}$ and concentrated under reduced pressure. The residue was suspended in anhydrous acetonitrile $(2 \mathrm{~mL})$, and TBAF $(0.32 \mathrm{~g}, 1.00$ mmol) was added to the suspension. The reaction mixture was stirred at room temperature for $10 \mathrm{~h}$ then concentrated under reduced pressure. The residue was chromatographed (silica gel, hexane/ethyl acetate in gradient 30 to $50 \%)$ to give the product $16 \mathrm{c}(0.26 \mathrm{~g}, 87 \%)$ as dark-brown oil; ${ }^{1} \mathrm{H}$ NMR (400 MHz, DMSO- $d_{6}$ ) $\delta$ 1.10-1.16 (m, 2H, cyclopropyl), 1.27-1.34 (m, 2H, cyclopropyl), 2.32 (s, 3H, Me), 2.41-2.48 (m, 1H, cyclopropyl), 2.74-2.84 (m, 4H, $\left.\mathrm{CH}_{2} \mathrm{CH}_{2}\right), 6.80$ (dd, 1H, J 7.9, $\left.1.2 \mathrm{~Hz}, \mathrm{C}_{6} \mathrm{H}_{4}\right), 7.10\left(\mathrm{ddd}, 1 \mathrm{H}, J 7.9,1.3 \mathrm{~Hz}, \mathrm{C}_{6} \mathrm{H}_{4}\right), 7.28$ (ddd, 1H, J 7.5, $1.2 \mathrm{~Hz}, \mathrm{C}_{6} \mathrm{H}_{4}$ ), 7.35 (dd, $\left.1 \mathrm{H}, J 7.4,1.3 \mathrm{~Hz}, \mathrm{C}_{6} \mathrm{H}_{4}\right), 8.56(\mathrm{~s}, 1 \mathrm{H}, 6-\mathrm{H})$. APCI MS $m / z: 304[\mathrm{M}+\mathrm{H}]^{+}$. Anal. Calcd for $\mathrm{C}_{19} \mathrm{H}_{17} \mathrm{~N}_{3} \mathrm{O}$ (303.37): C, 75.23; H, 5.65; N, 13.85\%. Found: C, 75.20; H, 5.54; N, 13.75\%. 
3-(5-tert-Butyl-1,2,4-oxadiazol-3-yl)-2-methyl-4-phenylpyridine (16d). Pivaloyl chloride (0.11 g, $0.93 \mathrm{mmol})$ was added at $0{ }^{\circ} \mathrm{C}$ to solution of $N$-hydroxy-2-methyl-4-phenylpyridine-3carboximidamide 13d $(0.20 \mathrm{~g}, 0.88 \mathrm{mmol})$ and triethylamine $(0.1 \mathrm{~g}, 0.14 \mathrm{~mL}, 0.97 \mathrm{mmol})$ in anhydrous dichloromethane $(2 \mathrm{~mL})$ under stirring. The reaction mixture was stirred at room temperature for $3 \mathrm{~h}$ then poured into saturated aqueous solution of $\mathrm{NaHCO}_{3}(5 \mathrm{~mL})$. The product was extracted with ethyl acetate $(4 \times 5 \mathrm{~mL})$, and the extract was dried over $\mathrm{MgSO}_{4}$ and concentrated under reduced pressure. The residue was suspended in anhydrous acetonitrile $(3 \mathrm{~mL})$, and TBAF (0.28 g, $0.88 \mathrm{mmol})$ was added to the suspension. The reaction mixture was stirred at room temperature for $10 \mathrm{~h}$ then concentrated under reduced pressure. The residue was chromatographed (silica gel, hexane/ethyl acetate in gradient 30 to $50 \%$ ) to give the product $\mathbf{1 6 d}$ $(0.21 \mathrm{~g}, 81 \%)$ as yellow oil; ${ }^{1} \mathrm{H} \mathrm{NMR}\left(400 \mathrm{MHz}, \mathrm{DMSO}-d_{6}\right) \delta 1.32(\mathrm{~s}, 9 \mathrm{H}, t$-Bu), $2.42(\mathrm{~s}, 3 \mathrm{H}, \mathrm{Me})$, 7.15-7.20 (m, 2H, Ph), 7.32-7.35 (m, 3H, Ph), 7.41 (d, 1H, J 5.1 Hz, 5-H), 8.68 (d, 1H, J 5.1 Hz, 6H). APCI MS $m / z$ : $294[\mathrm{M}+\mathrm{H}]^{+}$. Anal. Calcd for $\mathrm{C}_{18} \mathrm{H}_{19} \mathrm{~N}_{3} \mathrm{O}$ (293.37): C, 73.70; H, 6.53; N, 14.32\%. Found: C, 73.58; H, 6.51; N, 14.14\%.

Methyl 3-(2-methyl-4-phenylpyridin-3-yl)-1,2,4-oxadiazole-5-carboxylate (16e). Methyl oxalyl chloride $(0.113 \mathrm{~g}, 0.92 \mathrm{mmol})$ was added at $0{ }^{\circ} \mathrm{C}$ to solution of $N$-hydroxy-2-methyl-4phenylpyridine-3-carboximidamide $13 d(0.20 \mathrm{~g}, 0.88 \mathrm{mmol})$ and triethylamine $(0.1 \mathrm{~g}, 0.14 \mathrm{~mL}$, $0.97 \mathrm{mmol})$ in anhydrous dichloromethane $(2 \mathrm{~mL})$ under stirring. The reaction mixture was stirred at room temperature for $12 \mathrm{~h}$ then poured into saturated aqueous solution of $\mathrm{NaHCO}_{3}(5 \mathrm{~mL})$. The product was extracted with ethyl acetate $(4 \times 5 \mathrm{~mL})$, and the extract was dried over $\mathrm{MgSO}_{4}$ and concentrated under reduced pressure. The residue was suspended in anhydrous acetonitrile $(3 \mathrm{~mL})$, and TBAF $(0.28 \mathrm{~g}, 0.88 \mathrm{mmol})$ was added to the suspension. The reaction mixture was stirred at room temperature for $10 \mathrm{~h}$ then concentrated under reduced pressure. The residue was chromatographed (silica gel, hexane/ethyl acetate in gradient 30 to $50 \%$ ) to give the product $\mathbf{1 6 e}$ $(0.12 \mathrm{~g}, 46 \%)$ as pale-yellow solid; mp 114-117 ${ }^{\circ} \mathrm{C} ;{ }^{1} \mathrm{H}$ NMR (400 MHz, DMSO- $\left.d_{6}\right) \delta 2.41$ (s, 3H, $\mathrm{Me}$ ), 3.96 (s, 3H, MeO), 7.15-7.22 (m, 2H, Ph), 7.32-7.39 (m, 3H, Ph), 7.44 (d, 1H, J 5.0 Hz, 5-H), $8.72(\mathrm{~d}, 1 \mathrm{H}, J 5.0 \mathrm{~Hz}, 6-\mathrm{H})$. APCI MS m/z: $296[\mathrm{M}+\mathrm{H}]^{+}$. Anal. Calcd for $\mathrm{C}_{16} \mathrm{H}_{13} \mathrm{~N}_{3} \mathrm{O}_{3}(295.30)$ : C, $65.08 ; \mathrm{H}, 4.44 ; \mathrm{N}, 14.23 \%$. Found: C, 65.11; H, 4.47; N, 14.13\%.

2-Methyl-1-[5-(trifluoromethyl)-1,2,4-oxadiazol-3-yl]-5,6-dihydrobenzo[f]isoquinoline (16f). Trifluoroacetic anhydride $(0.25 \mathrm{~g}, 0.17 \mathrm{~mL}, 1.2 \mathrm{mmol})$ was added dropwise to solution of $N$ hydroxy-2-methyl-5,6-dihydrobenzo[f]isoquinoline-1-carboximidamide 13c $(0.10 \mathrm{~g}, 0.4 \mathrm{mmol})$ in anhydrous acetonitrile $(2 \mathrm{~mL})$ under stirring. The reaction mixture was stirred at room temperature for $12 \mathrm{~h}$ then diluted with saturated aqueous solution of $\mathrm{NaHCO}_{3}(5 \mathrm{~mL})$. The product was extracted with ethyl acetate $(4 \times 5 \mathrm{~mL})$, and the extract was dried over $\mathrm{MgSO}_{4}$ and concentrated under reduced pressure. The residue was chromatographed (silica gel, hexane/ethyl acetate in gradient 30 to $50 \%)$ to give the product $\mathbf{1 6 f}(0.13 \mathrm{~g}, 98 \%)$ as white solid; mp $105-106{ }^{\circ} \mathrm{C} ;{ }^{1} \mathrm{H} \mathrm{NMR}(400 \mathrm{MHz}$, DMSO-d $\left.d_{6}\right) 2.43(\mathrm{~s}, 3 \mathrm{H}, \mathrm{Me}), 2.76-2.87\left(\mathrm{~m}, 4 \mathrm{H}, \mathrm{CH}_{2} \mathrm{CH}_{2}\right), 6.66\left(\mathrm{dd}, 1 \mathrm{H}, J 7.9,1.2 \mathrm{~Hz}, \mathrm{C}_{6} \mathrm{H}_{4}\right), 7.10$ (ddd, 1H, J 7.9, $1.3 \mathrm{~Hz}, \mathrm{C}_{6} \mathrm{H}_{4}$ ), 7.30 (ddd, 1H, J 7.4, $1.2 \mathrm{~Hz}, \mathrm{C}_{6} \mathrm{H}_{4}$ ), 7.38 (dd, 1H, J 7.4, $1.3 \mathrm{~Hz}$, $\left.\mathrm{C}_{6} \mathrm{H}_{4}\right), 8.64(\mathrm{~s}, 1 \mathrm{H}, 6-\mathrm{H})$. APCI MS $m / z: 332[\mathrm{M}+\mathrm{H}]^{+}$. Anal. Calcd for $\mathrm{C}_{17} \mathrm{H}_{12} \mathrm{~F}_{3} \mathrm{~N}_{3} \mathrm{O}(331.30): \mathrm{C}$, 61.63; H, 3.65; F, 17.20; N, 12.68\%. Found: C, 61.67; H, 3.64; F, 17.09; N, 12.64\%. 
2-Methyl-N,4-diphenylpyridine-3-carboximidamide hydrochloride (17a). $2 \mathrm{M}$ solution of trimethylaluminum in toluene $(10 \mathrm{~mL}, 20 \mathrm{mmol})$ was slowly added dropwise to solution of aniline $(1.4 \mathrm{~g}, 15 \mathrm{mmol})$ in anhydrous dichloroethane $(10 \mathrm{~mL})$ under stirring at $0{ }^{\circ} \mathrm{C}$ in inert atmosphere (argon). The mixture was stirred at room temperature for $30 \mathrm{~min}$ then solution of 2-methyl-4phenylnicotinonitrile $1 \mathbf{d}(1.94 \mathrm{~g}, 10 \mathrm{mmol})$ in anhydrous dichloroethane $(10 \mathrm{~mL})$ was added under stirring. The reaction mixture formed was subjected to microwave irradiation at $130{ }^{\circ} \mathrm{C}$ for $3 \mathrm{~h}$ then cooled to room temperature and carefully treated with methanol $(100 \mathrm{~mL})$. The jelly-like precipitate was filtered off through Celite and washed with methanol. The filtrate was concentrated under reduced pressure, and the residue was chromatographed (silica gel, chloroform/methanol in gradient 0 to $30 \%$ ) to give the viscous unstable oil that was identified as hydrochloride. For that, the oily compound was dissolved in anhydrous dioxane $(15 \mathrm{~mL})$, and $4 \mathrm{M}$ solution of $\mathrm{HCl}$ in dioxane $(7.5$ $\mathrm{mL}, 3$ eq.) was added under stirring. The precipitate formed was filtered off, washed with anhydrous ether and dried in vacuum at room temperature to afford the titled compound 17a (2.65 g, 82\%) as pale-yellow solid; mp $214-216{ }^{\circ} \mathrm{C} ;{ }^{1} \mathrm{H}$ NMR (400 MHz, DMSO- $\left.d_{6}\right) \delta 2.82$ (s, 3H, Me), 7.09-7.14 (m, 2H, Ph), 7.35-7.65 (m, 8H, Ph), 7.71 (d, 1H, J 5.4 Hz, 5-H), 8.87 (d, 1H, J 5.4 Hz, 6H), 9.36 (br. s, 1H, NH), 10.44 (br. s, 1H, NH), 12.69 (br. s, 1H, NH). IR (film) $v 3424$ (NH), 3247 $(=\mathrm{NH}), 1631(\mathrm{C}=\mathrm{N}) \mathrm{cm}^{-1}$. APCI MS m/z: $288[\mathrm{M}+\mathrm{H}]^{+}$. Anal. Calcd for $\mathrm{C}_{19} \mathrm{H}_{18} \mathrm{ClN}_{3}(323.83)$ : C, 70.47; H, 5.60; Cl, 10.95; N, 12.98\%. Found: C, 70.33; H, 5.61; Cl, 11.02; N, 12.79\%.

3-Methyl- $N$-propyl-6,7-dihydro-5H-cyclopenta[c]pyridine-4-carboximidamide dihydrochloride (17b). Obtained analogously to previous amidine from 3-methyl-6,7-dihydro-5Hcyclopenta[c]pyridine-4-carbonitrile 1a and propylamine, yield 66\%, colorless solid; mp 207-209 ${ }^{\circ} \mathrm{C}$ (decomp.); ${ }^{1} \mathrm{H}$ NMR (400 MHz, DMSO-d 6$) \delta 0.97$ (t, 3H, J 7.0 Hz, $\left.\mathrm{CH}_{2} \mathrm{CH}_{2} \mathrm{CH}_{3}\right), 1.61-1.72(\mathrm{~m}$, $\left.2 \mathrm{H}, \mathrm{CH}_{2} \mathrm{CH}_{2} \mathrm{CH}_{3}\right), 2.10-2.21\left(\mathrm{~m}, 2 \mathrm{H}, \mathrm{CH}_{2}\right.$ of carbocycle), 2.67 (s, 3H, 2-Me), 3.01-3.10 (m, 4H, $\mathrm{CH}_{2} \mathrm{CH}_{2}$ of carbocycle), 3.44 (dt, $\left.2 \mathrm{H}, J 7.0 \mathrm{~Hz}, \mathrm{CH}_{2} \mathrm{CH}_{2} \mathrm{CH}_{3}\right), 8.71$ (s, $\left.1 \mathrm{H}, 6-\mathrm{H}\right), 9.75$ (br. s, $1 \mathrm{H}$, $\mathrm{NH}), 9.96$ (br. s, 1H, NH), 10.56 (br. s, 1H, NH). IR (film) v $3553(\mathrm{NH}), 3466(=\mathrm{NH}), 2985(\mathrm{CH})$, $1684(\mathrm{C}=\mathrm{N}) \mathrm{cm}^{-1}$. APCI MS m/z: $218[\mathrm{M}+\mathrm{H}]^{+}$. Anal. Calcd for $\mathrm{C}_{13} \mathrm{H}_{21} \mathrm{Cl}_{2} \mathrm{~N}_{3}$ (290.24): C, 53.80; $\mathrm{H}$, 7.29; Cl, 24.43; N, 14.48\%. Found: C, 53.73; H, 7.30; Cl, 24.35; N, 14.40\%.

3-(4,5-Dimethyl-1-phenyl-1H-imidazol-2-yl)-2-methyl-4-phenylpyridine (19a). Sodium carbonate $(0.74 \mathrm{~g}, 7.0 \mathrm{mmol})$ then 3-chlorobutan-2-one $18(0.37 \mathrm{~g}, 3.5 \mathrm{mmol})$ were added to solution of amidine $17 \mathbf{a}$ (free base) $(0.50 \mathrm{~g}, 1.74 \mathrm{mmol})$ in anhydrous ethanol $(10 \mathrm{~mL})$. The resulting mixture was subjected to microwave irradiation at $130{ }^{\circ} \mathrm{C}$ for $3 \mathrm{~h}$. Additional amount of 3-chlorobutan-2-one $(0.37 \mathrm{~g}, 3.5 \mathrm{mmol})$ was added to the mixture, and the microwave irradiation was continued under the same conditions for $3 \mathrm{~h}$. The reaction mass was cooled, filtered and concentrated under reduced pressure. The residue was chromatographed (silica gel, chloroform/methanol 95:5) to give the product 19a (0.40 g, 67\%) as pale-yellow oil; ${ }^{1} \mathrm{H} \mathrm{NMR}\left(400 \mathrm{MHz}, \mathrm{CDCl}_{3}\right) \delta 1.87$ (s, 3H, Me), 2.32 (s, 3H, Me), 2.65 (s, 3H, Me), 6.11-6.21 (m, 2H, Ph), 6.82-6.87 (m, 2H, Ph), 6.93 (d, 1H, J 5.1 Hz, 5-H), 6.98-7.04 (m, 2H, Ph), 7.09-7.15 (m, 1H, Ph), 7.16-7.22 (m, 2H, Ph), 7.24-7.30 (m, 1H, Ph), 8.44 (d, $1 \mathrm{H}, J 5.1 \mathrm{~Hz}, 6-\mathrm{H})$. APCI MS m/z: $340[\mathrm{M}+\mathrm{H}]^{+}$. Anal. Calcd for $\mathrm{C}_{23} \mathrm{H}_{21} \mathrm{~N}_{3}(339.44)$ : C, 81.39; H, 6.24; N, 12.38\%. Found: C, 81.25; H, 6.08; N, 12.31\%.

4-(4,5-Dimethyl-1-propyl-1H-imidazol-2-yl)-3-methyl-6,7-dihydro-5H-cyclopenta[c]pyridine (19b). Prepared analogously from amidine 17b (free base) and 3-chlorobutan-2-one 18; glassy 
mass; yield $0.57 \mathrm{~g}(92 \%) ;{ }^{1} \mathrm{H}$ NMR (400 MHz, DMSO-d 6 ) $\delta 0.66\left(\mathrm{t}, 3 \mathrm{H}, J 7.3 \mathrm{~Hz}, \mathrm{CH}_{2} \mathrm{CH}_{2} \mathrm{CH}_{3}\right.$ ), 1.31-1.42 (m, 2H, $\left.\mathrm{CH}_{2} \mathrm{CH}_{2} \mathrm{CH}_{3}\right)$, 1.94-2.03 (m, 2H, $\mathrm{CH}_{2}$ of carbocycle), 2.08 (s, 3H, 2-Me), 2.16 (s, $3 \mathrm{H}, \mathrm{Me}), 2.18(\mathrm{~s}, 3 \mathrm{H}, \mathrm{Me}), 2.46-2.56\left(\mathrm{~m}, 1 \mathrm{H}, \mathrm{CH}_{2}\right.$ of carbocycle), 2.58-2.68 (m, $1 \mathrm{H}, \mathrm{CH}_{2}$ of carbocycle), $2.92\left(\mathrm{~d}, 2 \mathrm{H}, J 7.3 \mathrm{~Hz}, \mathrm{CH}_{2}\right.$ of carbocycle), $3.52\left(\mathrm{t}, 2 \mathrm{H}, J 7.1 \mathrm{~Hz}, \mathrm{CH}_{2} \mathrm{CH}_{2} \mathrm{CH}_{3}\right), 8.37$ (s, 1H, 6-H). APCI MS m/z: $270[\mathrm{M}+\mathrm{H}]^{+}$. Anal. Calcd for $\mathrm{C}_{17} \mathrm{H}_{23} \mathrm{~N}_{3}$ (269.39): C, 75.80; H, 8.61; N, $15.60 \%$. Found: C, 76.03; H, 8.80; N, $15.41 \%$.

2-Methyl-4-phenyl-3-[1-phenyl-4-(trifluoromethyl)-1H-imidazol-2-yl]pyridine (22). A mixture of 2-methyl- $N$,4-diphenylpyridine-3-carboximidamide $17 \mathbf{a}(0.5 \mathrm{~g}, 1.74 \mathrm{mmol}), \mathrm{NaHCO}_{3}(0.3 \mathrm{~g}, 3.6$ $\mathrm{mmol})$ and 3-bromo-1,1,1-trifluoroacetone $20(0.69 \mathrm{~g}, 3.6 \mathrm{mmol})$ in anhydrous ethanol $(10 \mathrm{~mL})$ was heated under stirring at $75{ }^{\circ} \mathrm{C}$ for $3 \mathrm{~h}$ then cooled and filtered. The filtrate was concentrated under reduced pressure. The residue was chromatographed (silica gel, hexane/ethyl acetate in gradient 50 to $100 \%)$ to furnish intermediate 2-(2-methyl-4-phenylpyridin-3-yl)-1-phenyl-4(trifluoromethyl)-4,5-dihydro-1H-imidazol-4-ol (21) (0.55 g, 80\%), mp 94-95 ${ }^{\circ} \mathrm{C}$. The ${ }^{1} \mathrm{H}$ NMR spectrum was a superposition of two ones: (a), 57\%; ${ }^{1} \mathrm{H}$ NMR (400 MHz, DMSO- $\left.d_{6}\right) \delta 2.71(\mathrm{~s}, 3 \mathrm{H}$, Me), 3.21-3.60 (br. s, 1H, OH), 3.68 (d, 1H, J $\left.12.0 \mathrm{~Hz}, \mathrm{CH}_{2}\right), 3.96$ (d, 1H, J $12.0 \mathrm{~Hz}, \mathrm{CH}_{2}$ ), 6.216.26 (m, 2H, Ph), 6.89-7.03 (m, 4H, Ph), 7.12-7.41 (m, 5H, Ph, 5-H), 8.59 (d, 1H, J 5.1 Hz, 6-H); (b), 43\%; ${ }^{1} \mathrm{H}$ NMR (400 MHz, DMSO- $\left.d_{6}\right) \delta 2.58$ (s, 3H, Me), 3.20-3.59 (br. s, 1H, OH), 3.79 (d, $\left.1 \mathrm{H}, J 12.0 \mathrm{~Hz}, \mathrm{CH}_{2}\right), 4.02$ (d, 1H, J 12.0 Hz, $\mathrm{CH}_{2}$ ), 6.21-6.26 (m, 2H, Ph), 6.89-7.03 (m, 4H, Ph), 7.12-7.41 (m, 5H, Ph, 5-H), 8.58 (d, 1H, J 5.1 Hz, 6-H). The intermediate alcohol 21 (0.48 g, 1.2 $\mathrm{mmol})$ was dissolved in anhydrous dichloromethane $(10 \mathrm{~mL})$, and trifluoroacetic anhydride $(2 \mathrm{~mL})$ was added to the solution. The reaction mixture was stirred at room temperature for $30 \mathrm{~min}$ then evaporated to dryness. The residue was mixed with saturated aqueous solution of $\mathrm{NaHCO}_{3}(10 \mathrm{~mL})$ then treated with ethyl acetate $(4 \times 10 \mathrm{~mL})$. The extract was dried over $\mathrm{MgSO}_{4}$ and concentrated under reduced pressure. The residue was chromatographed (silica gel, hexane/ethyl acetate in gradient 30 to $50 \%)$ to afford the product $22(0.43 \mathrm{~g}, 94 \%)$ as pale-yellow solid; mp $133-134{ }^{\circ} \mathrm{C}$ (from $\mathrm{Et}_{2} \mathrm{O}$ ); ${ }^{1} \mathrm{H}$ NMR (400 MHz, DMSO-d $) \delta 2.53$ (s, 3H, Me), 6.45 (d, 2H, J 7.3 Hz, Ph), 6.68 (d, $2 \mathrm{H}, J 7.3 \mathrm{~Hz}, \mathrm{Ph}), 7.13-7.35$ (m, 6H, Ph), 7.18 (d, 1H, J 5.2 Hz, 5-H), 8.13 (s, 1H, CH of imidazole ring), $8.78(\mathrm{~d}, 1 \mathrm{H}, J 5.2 \mathrm{~Hz}, 6-\mathrm{H}) .{ }^{13} \mathrm{C}$ NMR (100 MHz, DMSO-d 6 ) $\delta 23.2(7-\mathrm{C}), 121.5$ (5-C), 121.9 (17-C), 122.0 (18-C), 122.7 (3-C), 124.1 (21-C, 25-C), 127.9 (9-C, 13-C), 128.2 (11-C), 128.3 (23C), 128.4 (10-C, 12-C), 129.0 (22-C, 24-C), 130.6 (16-C), 135.2 (20-C), 137.2 (4-C), 144.3 (14-C), 149.3 (8-C), 150.3 (6-C), 158.8 (2-C) (atom numbering see Figure 2). APCI MS m/z: $380[\mathrm{M}+\mathrm{H}]^{+}$. Anal. Calcd for $\mathrm{C}_{22} \mathrm{H}_{16} \mathrm{~F}_{3} \mathrm{~N}_{3}$ (379.39): C, 69.65; H, 4.25; F, 15.02; N, 11.08\%. Found: C, 69.70; H, 4.43 ; F, 15.06; N, 11.00\%.

3-Methyl-6,7-dihydro-5H-cyclopenta[c]pyridine-4-carbothioamide (23a). 3-Methyl-6,7dihydro-5H-cyclopenta[c]pyridine-4-carbonitrile $\mathbf{1 a}(0.79 \mathrm{~g}, 5 \mathrm{mmol})$ was suspended in water (3 $\mathrm{mL})$, and conc. $\mathrm{HCl}(1 \mathrm{~mL}, 10 \mathrm{mmol})$ then $O, O$-diethyl dithiophosphate $(1.86 \mathrm{~g}, 1.68 \mathrm{~mL}, 10 \mathrm{mmol})$ were successively added dropwise to the suspension under stirring at room temperature. The resulting mixture was stirred at $80{ }^{\circ} \mathrm{C}$ for $8 \mathrm{~h}$ (TLC monitoring, silica gel, ethyl acetate). The reaction mixture was then cooled to room temperature and slowly poured into stirred saturated aqueous solution of $\mathrm{NaHCO}_{3}(30 \mathrm{~mL})$. The mixture was extracted with ethyl acetate $(4 \times 25 \mathrm{~mL})$, and the extract was washed with brine $(10 \mathrm{~mL})$, dried over $\mathrm{MgSO}_{4}$ and evaporated at $60{ }^{\circ} \mathrm{C} / 20$ Torr. 
The residue was recrystallized from ethyl acetate to give the product 23a $(0.62 \mathrm{~g}, 65 \%)$ as palebrown powder; mp $220{ }^{\circ} \mathrm{C}$ (subliming); ${ }^{1} \mathrm{H}$ NMR (400 MHz, DMSO- $\left.d_{6}\right) \delta 1.97-2.07$ (m, 2H, $\mathrm{CH}_{2}$ ), 2.42 (s, 3H, Me), $2.81\left(\mathrm{t}, 2 \mathrm{H}, J 7.5 \mathrm{~Hz}, \mathrm{CH}_{2}\right), 2.88$ (t, 2H,J 7.5 Hz, $\left.\mathrm{CH}_{2}\right), 8.24(\mathrm{~s}, 1 \mathrm{H}, 6-\mathrm{H}), 9.61$ and 10.07 (both br. s, $1 \mathrm{H}$ and 1H, $\mathrm{NH}_{2}$ ). IR (film) $v 3204(\mathrm{NH}), 2991(\mathrm{CH}), 1181(\mathrm{C}=\mathrm{S}) \mathrm{cm}^{-1}$. APCI MS $m / z$ : $193[\mathrm{M}+\mathrm{H}]^{+}$. Anal. Calcd for $\mathrm{C}_{10} \mathrm{H}_{12} \mathrm{~N}_{2} \mathrm{~S}$ (192.28): C, 62.47; H, 6.29; N, 14.57; S, 16.68\%. Found: C, 62.45; H, 6.38; N, 14.45; S, 16.69\%.

The mother liquid after recrystallization was chromatographed (silica gel, ethyl acetate) to afford the starting nitrile $\mathbf{1 a}(0.17 \mathrm{~g}, 21 \%)$.

3-Methyl-6,7,8,9-tetrahydro-5H-cyclohepta[c]pyridine-4-carbothioamide (23b). Prepared analogously from 3-methyl-6,7,8,9-tetrahydro-5H-cyclohepta[c]pyridine-4-carbonitrile $\mathbf{1 b}(0.93 \mathrm{~g}$, $5 \mathrm{mmol}$ ); reaction time $20 \mathrm{~h}$; the crude product was chromatographed (silica gel, hexane/ethyl acetate in gradient 50 to $100 \%)$ to give the starting nitrile $\mathbf{1 b}(0.16 \mathrm{~g}, 17 \%)$ and the target product 23b $(0.49 \mathrm{~g}, 45 \%)$ as pale-yellow powder; mp 238-239 ${ }^{\circ} \mathrm{C}$ (from EtOH); ${ }^{1} \mathrm{H}$ NMR (400 MHz, DMSO- $\left.d_{6}\right) \delta 1.46-1.65\left(\mathrm{~m}, 4 \mathrm{H}, \mathrm{CH}_{2} \mathrm{CH}_{2}\right), 1.74-1.85\left(\mathrm{~m}, 2 \mathrm{H}, \mathrm{CH}_{2}\right), 2.39(\mathrm{~s}, 3 \mathrm{H}, \mathrm{Me}), 2.68-2.78(\mathrm{~m}$, 4H, $2 \mathrm{CH}_{2}$ ), 8.11 (s, 1H, 6-H), 9.66 and 10.12 (both br. s, $1 \mathrm{H}$ and $1 \mathrm{H}, \mathrm{NH}_{2}$ ). IR (film) v 3209 (NH), $2937(\mathrm{CH}), 1162(\mathrm{C}=\mathrm{S}) \mathrm{cm}^{-1}$. APCI MS m/z: $221[\mathrm{M}+\mathrm{H}]^{+}$. Anal. Calcd for $\mathrm{C}_{12} \mathrm{H}_{16} \mathrm{~N}_{2} \mathrm{~S}(220.34)$ : C, 65.41; H, 7.32; N, 12.71; S, 14.55\%. Found: C, 65.52; H, 7.41; N, 12.58; S, 14.40\%.

2-Methyl-5,6-dihydrobenzo[f]isoquinoline-1-carbothioamide (23c). Prepared analogously from 2-methyl-5,6-dihydrobenzo[f]isoquinoline-1-carbonitrile 1c $(1.10 \mathrm{~g}, 5 \mathrm{mmol})$; additional amount of $O, O$-diethyl dithiophosphate $(0.93 \mathrm{~g}, 0.84 \mathrm{~mL}, 5 \mathrm{mmol})$ was added to reaction mixture after heating at $80{ }^{\circ} \mathrm{C}$ for $95 \mathrm{~h}$, and the resulting mixture was additionally heated under the same conditions for 5 h. The crude mass after work-up was recrystallized from ethyl acetate to furnish the pure product 23c $(0.79 \mathrm{~g}, 62 \%)$ as pale-yellow crystal solid; mp $225-227{ }^{\circ} \mathrm{C} ;{ }^{1} \mathrm{H}$ NMR (400 MHz, DMSO- $\left.d_{6}\right) \delta$ 2.53 (s, 3H, Me), 2.64-2.80 (m, 4H, $\left.\mathrm{CH}_{2} \mathrm{CH}_{2}\right), 7.21-7.35\left(\mathrm{~m}, 3 \mathrm{H}, \mathrm{C}_{6} \mathrm{H}_{4}\right), 8.33$ (s, 1H, 6-H), 8.35$8.41\left(\mathrm{~m}, 1 \mathrm{H}, \mathrm{C}_{6} \mathrm{H}_{4}\right), 9.93$ and 10.29 (both br. s, $1 \mathrm{H}$ and $1 \mathrm{H}, \mathrm{NH}_{2}$ ). IR (film) $v 3396(\mathrm{NH}), 2946$ $(\mathrm{CH}), 1159(\mathrm{C}=\mathrm{S}) \mathrm{cm}^{-1}$. APCI MS $m / z: 255[\mathrm{M}+\mathrm{H}]^{+}$. Anal. Calcd for $\mathrm{C}_{15} \mathrm{H}_{14} \mathrm{~N}_{2} \mathrm{~S}$ (254.36): C, 70.83; H, 5.55; N, 11.01; S, 12.61\%. Found: C, 70.83; H, 5.43; N, 11.10; S, 12.46\%.

2-Methyl-4-phenylpyridine-3-carbothioamide (23d). Prepared analogously to thioamide 23a from 2-methyl-4-phenylnicotinonitrile $\mathbf{1 d}(0.97 \mathrm{~g}, 5 \mathrm{mmol})$; the crude product was recrystallized from ethyl acetate to give the product $23 d(0.97 \mathrm{~g}, 85 \%)$ as yellow powder; mp 199-200 ${ }^{\circ} \mathrm{C} ;{ }^{1} \mathrm{H}$ NMR (400 MHz, DMSO-d $d_{6} \delta 2.55$ (s, 3H, Me), 7.15 (d, 1H, J 5.0 Hz, 5-H), 7.36-7.45 (m, 3H, Ph), 7.48-7.54 (m, 2H, Ph), $8.43(\mathrm{~d}, 1 \mathrm{H}, J 5.0 \mathrm{~Hz}, 6-\mathrm{H}), 9.76$ and 10.03 (both br. s, $1 \mathrm{H}$ and $1 \mathrm{H}, \mathrm{NH}_{2}$ ). IR (film) $v 3097(\mathrm{NH}), 1110(\mathrm{C}=\mathrm{S}) \mathrm{cm}^{-1}$. APCI MS m/z: $229[\mathrm{M}+\mathrm{H}]^{+}$. Anal. Calcd for $\mathrm{C}_{13} \mathrm{H}_{12} \mathrm{~N}_{2} \mathrm{~S}$ (228.32): C, 68.39; H, 5.30; N, 12.27; S, 14.04\%. Found: C, 68.19; H, 5.38; N, 12.29; S, 14.02\%. The starting nitrile $1 \mathbf{d}(0.09 \mathrm{~g}, 10 \%)$ was isolated from the mother liquid.

4-tert-Butyl-2-methylpyridine-3-carbothioamide (23e). Prepared analogously to thioamide 23c from 4-tert-butyl-2-methylnicotinonitrile $1 \mathrm{e}(0.88 \mathrm{~g}, 5 \mathrm{mmol})$; the crude product was chromatographed (silica gel, hexane/ethyl acetate in gradient 50 to $100 \%$ ) to give the starting nitrile 1e $(0.52 \mathrm{~g}, 59 \%)$ and the product $23 \mathrm{e}(0.41 \mathrm{~g}, 39 \%)$ as white crystal solid; mp $196-197{ }^{\circ} \mathrm{C}$ (from benzene); ${ }^{1} \mathrm{H}$ NMR (400 MHz, DMSO- $\left.d_{6}\right) \delta 1.45$ (s, 9H, $t$-Bu), $2.48(\mathrm{~s}, 3 \mathrm{H}, \mathrm{Me}), 7.28(\mathrm{~d}, 1 \mathrm{H}, J 5.6$ $\mathrm{Hz}, 5-\mathrm{H}), 8.27$ (d, 1H, J 5.6 Hz, 6-H), 9.80 and 10.21 (both br. s, $1 \mathrm{H}$ and $1 \mathrm{H}, \mathrm{NH}_{2}$ ). IR (film) $v 3225$ 
$(\mathrm{NH}), 2969(\mathrm{CH}), 1195(\mathrm{C}=\mathrm{S}) \mathrm{cm}^{-1}$. APCI MS m/z: $209[\mathrm{M}+\mathrm{H}]^{+}$. Anal. Calcd for $\mathrm{C}_{11} \mathrm{H}_{16} \mathrm{~N}_{2} \mathrm{~S}$ (208.33): C, 63.42; H, 7.74; N, 13.45; S, 15.39\%. Found: C, 63.48; H, 7.73; N, 13.33; S, 15.50\%.

4-Isopropyl-2-methylpyridine-3-carbothioamide (23f). Prepared analogously to thioamide 23a from 4-isopropyl-2-methylnicotinonitrile $\mathbf{1 f}(0.80 \mathrm{~g}, 5 \mathrm{mmol})$; the crude product obtained after heating at $80{ }^{\circ} \mathrm{C}$ for $40 \mathrm{~h}$ was recrystallized from ethyl acetate to give the product $\mathbf{2 3 f}(0.66 \mathrm{~g}, 68 \%)$ as pale-yellow powder; mp 197-198 ${ }^{\circ} \mathrm{C}$ (from EtOH); ${ }^{1} \mathrm{H}$ NMR (400 MHz, DMSO- $\left.d_{6}\right) \delta 1.19(\mathrm{~d}$, $\left.6 \mathrm{H}, J 6.8 \mathrm{~Hz}, \mathrm{CH}\left(\mathrm{CH}_{3}\right)_{2}\right), 2.44$ (s, 3H, Me), 3.03-3.16 (septet, $\left.1 \mathrm{H}, J 6.8 \mathrm{~Hz}, \mathrm{CH}\left(\mathrm{CH}_{3}\right)_{2}\right), 7.20$ (d, $1 \mathrm{H}, J 5.4 \mathrm{~Hz}, 5-\mathrm{H}), 8.31$ (d, 1H, J $5.4 \mathrm{~Hz}, 6-\mathrm{H}), 9.71$ and 10.19 (both br. s, $1 \mathrm{H}$ and $1 \mathrm{H}, \mathrm{NH}_{2}$ ). IR (film) $v 3298(\mathrm{NH}), 2972(\mathrm{CH}), 1101(\mathrm{C}=\mathrm{S}) \mathrm{cm}^{-1}$. APCI MS m/z: $195[\mathrm{M}+\mathrm{H}]^{+}$. Anal. Calcd for $\mathrm{C}_{10} \mathrm{H}_{14} \mathrm{~N}_{2} \mathrm{~S}$ (194.30): C, 61.82; H, 7.26; N, 14.42; S, 16.50\%. Found: C, 61.80; H, 7.24; N, 14.43; S, $16.53 \%$. The starting nitrile $\mathbf{1 f}(0.24 \mathrm{~g}, 30 \%)$ was isolated from the mother liquid.

3-Methyl-5,6,7,8-tetrahydroisoquinoline-4-carbothioamide (23g). Prepared analogously to thioamide 23a from 3-methyl-5,6,7,8-tetrahydroisoquinoline-4-carbonitrile $1 \mathrm{~g}$ (0.86 g, $5 \mathrm{mmol})$; the crude product obtained after heating at $80{ }^{\circ} \mathrm{C}$ for $20 \mathrm{~h}$ was recrystallized from ethyl acetate to give the product $23 \mathrm{~g}(0.62 \mathrm{~g}, 60 \%)$ as pink powder; mp 235-236 ${ }^{\circ} \mathrm{C} ;{ }^{1} \mathrm{H}$ NMR (400 MHz, DMSO- $\left.d_{6}\right) \delta$ 1.63-1.77 (m, 4H, $\left.\mathrm{CH}_{2} \mathrm{CH}_{2}\right), 2.37$ (s, 3H, Me), 2.50-2.59 (m, 1H, $\left.\mathrm{CH}_{2}\right), 2.65-2.73\left(\mathrm{~m}, 2 \mathrm{H}, \mathrm{CH}_{2}\right)$, 2.78-2.89 (m, $\left.1 \mathrm{H}, \mathrm{CH}_{2}\right), 8.09(\mathrm{~s}, 1 \mathrm{H}, 6-\mathrm{H}), 9.61$ and 10.14 (both br. s, $1 \mathrm{H}$ and $1 \mathrm{H}, \mathrm{NH}_{2}$ ). IR (film) $v$ $3215(\mathrm{NH}), 2939(\mathrm{CH}), 1188(\mathrm{C}=\mathrm{S}) \mathrm{cm}^{-1}$. APCI MS m/z: $207[\mathrm{M}+\mathrm{H}]^{+}$. Anal. Calcd for $\mathrm{C}_{11} \mathrm{H}_{14} \mathrm{~N}_{2} \mathrm{~S}$ (206.31): C, 64.04; H, 6.84; N, 13.58; S, 15.54\%. Found: C, 63.98; H, 6.82; N, 13.34; S, 15.68\%. The starting nitrile $1 \mathrm{~g}(0.21 \mathrm{~g}, 25 \%)$ was isolated from the mother liquid.

3,5-Dimethyl-5,6,7,8-tetrahydroisoquinoline-4-carbothioamide (23h). Prepared analogously to thioamide 23c from 3,5-dimethyl-5,6,7,8-tetrahydroisoquinoline-4-carbonitrile $\mathbf{1 h}(0.93 \mathrm{~g}, 5 \mathrm{mmol})$; additional amount of $O, O$-diethyl dithiophosphate $(0.93 \mathrm{~g}, 0.84 \mathrm{~mL}, 5 \mathrm{mmol})$ was added to reaction mixture after heating at $80{ }^{\circ} \mathrm{C}$ for $10 \mathrm{~h}$, and the resulting mixture was additionally heated under the same conditions for $4 \mathrm{~h}$. The crude mass after work-up was chromatographed (silica gel, hexane/ethyl acetate in gradient 50 to $100 \%)$ to furnish the starting nitrile $\mathbf{1 h}(0.17 \mathrm{~g}, 18 \%)$ and the titled product $23 \mathrm{~h}(0.73 \mathrm{~g}, 66 \%)$ as pale-yellow crystal solid, mp $205-207{ }^{\circ} \mathrm{C}$ (from EtOH). The NMR spectrum of the product $\mathbf{2 3 h}$ was a superposition of two spectra corresponding to two rotamers: (a), 66\%; ${ }^{1} \mathrm{H}$ NMR (400 MHz, DMSO-d $) \delta 1.17$ (d, 3H, J 7.1 Hz, CH-CH $), 1.60-1.95$ (m, 4H, $\mathrm{CHCH}_{2} \mathrm{CH}_{2}$ ), 2.37 (s, 3H, 2-Me), 2.56-2.70 (m, 1H, $\left.\mathrm{CH}_{2}\right), 2.72-2.86\left(\mathrm{~m}, 1 \mathrm{H}, \mathrm{CH}_{2}\right), 3.50-$ $3.61\left(\mathrm{~m}, 1 \mathrm{H}, \mathrm{CH}-\mathrm{CH}_{3}\right), 8.10(\mathrm{~s}, 1 \mathrm{H}, 6-\mathrm{H}), 9.76$ and 10.20 (both br. s, $1 \mathrm{H}$ and $\left.1 \mathrm{H}, \mathrm{NH}_{2}\right) ;(\mathbf{b}), 34 \%$; ${ }^{1} \mathrm{H}$ NMR (400 MHz, DMSO- $\left.d_{6}\right) \delta 1.31\left(\mathrm{~d}, 3 \mathrm{H}, J 7.1 \mathrm{~Hz}, \mathrm{CH}-\mathrm{CH}_{3}\right), 1.60-1.95\left(\mathrm{~m}, 4 \mathrm{H}, \mathrm{CHCH}_{2} \mathrm{CH}_{2}\right)$, $2.40(\mathrm{~s}, 3 \mathrm{H}, 2-\mathrm{Me}), 2.56-2.70\left(\mathrm{~m}, 1 \mathrm{H}, \mathrm{CH}_{2}\right), 2.72-2.86\left(\mathrm{~m}, 1 \mathrm{H}, \mathrm{CH}_{2}\right), 2.99-3.08\left(\mathrm{~m}, 1 \mathrm{H}, \mathrm{CH}-\mathrm{CH}_{3}\right)$, $8.10(\mathrm{~s}, 1 \mathrm{H}, 6-\mathrm{H}), 9.57$ and 10.13 (both br. s, $1 \mathrm{H}$ and 1H, $\left.\mathrm{NH}_{2}\right)$. IR (film) v $3267(\mathrm{NH}), 2942(\mathrm{CH})$, $1179(\mathrm{C}=\mathrm{S}) \mathrm{cm}^{-1}$. APCI MS m/z: $221[\mathrm{M}+\mathrm{H}]^{+}$. Anal. Calcd for $\mathrm{C}_{12} \mathrm{H}_{16} \mathrm{~N}_{2} \mathrm{~S}$ (220.34): C, 65.41; $\mathrm{H}$, 7.32; N, 12.71; S, 14.55\%. Found: C, 65.37; H, 7.29; N, 12.81; S, 14.55\%.

2-Methyl-5H-chromeno[3,4-c]pyridine-1-carbothioamide (23i). Prepared analogously to thioamide 23c from 2-methyl-5H-chromeno[3,4-c]pyridine-1-carbonitrile 1i (1.11 g, $5 \mathrm{mmol})$; additional amount of $O, O$-diethyl dithiophosphate $(0.93 \mathrm{~g}, 0.84 \mathrm{~mL}, 5 \mathrm{mmol})$ was added to reaction mixture after heating at $80{ }^{\circ} \mathrm{C}$ for $30 \mathrm{~h}$, and the resulting mixture was additionally heated under the same conditions for $10 \mathrm{~h}$. The crude mass after work-up was recrystallized from ethyl acetate to 
give the product $23 \mathbf{i}(0.56 \mathrm{~g}, 44 \%)$ as pink-yellow crystal solid; mp $293-294{ }^{\circ} \mathrm{C} ;{ }^{1} \mathrm{H}$ NMR $(400$ $\left.\mathrm{MHz}, \mathrm{DMSO}-d_{6}\right) \delta 2.53(\mathrm{~s}, 3 \mathrm{H}, 2-\mathrm{Me}), 5.06\left(\mathrm{~s}, 2 \mathrm{H}, \mathrm{CH}_{2}\right), 7.03-7.08\left(\mathrm{~m}, 2 \mathrm{H}, \mathrm{C}_{6} \mathrm{H}_{4}\right), 7.34-7.40(\mathrm{~m}$, $\left.1 \mathrm{H}, \mathrm{C}_{6} \mathrm{H}_{4}\right), 8.36(\mathrm{~s}, 1 \mathrm{H}, 6-\mathrm{H}), 8.36-8.40\left(\mathrm{~m}, 1 \mathrm{H}, \mathrm{C}_{6} \mathrm{H}_{4}\right), 10.03$ and 10.41 (both br. s, $1 \mathrm{H}$ and $1 \mathrm{H}$, $\mathrm{NH}_{2}$ ). IR (film) v $3386(\mathrm{NH}), 3037(\mathrm{CH}), 1159(\mathrm{C}=\mathrm{S}) \mathrm{cm}^{-1}$. APCI MS m/z: $257[\mathrm{M}+\mathrm{H}]^{+}$. Anal. Calcd for $\mathrm{C}_{14} \mathrm{H}_{12} \mathrm{~N}_{2} \mathrm{OS}$ (256.33): C, 65.60; H, 4.72; N, 10.93; S, 12.51\%. Found: C, 65.46; H, 4.69; $\mathrm{N}, 11.10 ; \mathrm{S}, 12.46 \%$.

\section{4-[4-(4-Fluorophenyl)-1,3-thiazol-2-yl]-3-methyl-6,7-dihydro-5H-cyclopenta[c]pyridine}

hydrobromide (25a). The mixture of thioamide 23a $(0.10 \mathrm{~g}, 0.52 \mathrm{mmol})$, 2-bromo-1-(4fluorophenyl)ethanone $24 \mathrm{a}(0.12 \mathrm{~g}, 0.55 \mathrm{mmol})$ and anhydrous ethanol $(1 \mathrm{~mL})$ was stirred at $75{ }^{\circ} \mathrm{C}$ for $1 \mathrm{~h}$ then cooled. The precipitate was filtered off, washed with ethyl acetate $(2 \mathrm{~mL})$, and dried to afford the product 25a $(0.175 \mathrm{~g}, 86 \%)$ as pale-gray solid; mp 139-140 ${ }^{\circ} \mathrm{C}$ (decomp.); ${ }^{1} \mathrm{H}$ NMR (400 MHz, DMSO- $\left.d_{6}\right) \delta 2.13-2.23\left(\mathrm{~m}, 2 \mathrm{H}, \mathrm{CH}_{2}\right), 2.80(\mathrm{~s}, 3 \mathrm{H}, 2-\mathrm{Me}), 3.14\left(\mathrm{t}, 2 \mathrm{H}, J 7.6 \mathrm{~Hz}, \mathrm{CH}_{2}\right), 3.24(\mathrm{t}$, $\left.2 \mathrm{H}, J 7.6 \mathrm{~Hz}, \mathrm{CH}_{2}\right), 7.29-7.37\left(\mathrm{~m}, 2 \mathrm{H}, \mathrm{C}_{6} \mathrm{H}_{4}\right), 8.05-8.12\left(\mathrm{~m}, 2 \mathrm{H}, \mathrm{C}_{6} \mathrm{H}_{4}\right), 8.51(\mathrm{~s}, 1 \mathrm{H}, \mathrm{CH}$ of thiazole ring), 8.76 (s, $1 \mathrm{H}, \mathrm{CH}$ of pyridine ring). APCI MS m/z: $311[\mathrm{M}+\mathrm{H}]^{+}$. Anal. Calcd for $\mathrm{C}_{18} \mathrm{H}_{16} \mathrm{BrFN}_{2} \mathrm{~S}$ (391.31): C, 55.25; H, 4.12; Br, 20.42; N, 7.16; S, 8.19\%. Found: C, 55.17; H, 4.05; Br, 20.37; N, $7.11 ; \mathrm{S}, 8.35 \%$.

tert-Butyl 2-(3-methyl-6,7-dihydro-5H-cyclopenta[c]pyridin-4-yl)-6,7-dihydro[1,3]thiazolo[5,4-c]pyridine-5(4H)-carboxylate (25b). The mixture of thioamide 23a $(0.10 \mathrm{~g}, 0.52 \mathrm{mmol})$, tertbutyl 3-bromo-4-oxopiperidine-1-carboxylate $24 \mathbf{b}(0.15 \mathrm{~g}, 0.55 \mathrm{mmol}), \mathrm{NaHCO}_{3}(0.087 \mathrm{~g}, 1.04$ $\mathrm{mmol})$, and anhydrous ethanol $(2 \mathrm{~mL})$ was stirred at $70{ }^{\circ} \mathrm{C}$ for $24 \mathrm{~h}$ then cooled, diluted with ethyl acetate $(10 \mathrm{~mL})$ and filtered. The filtrate was concentrated under reduced pressure, and the residue was chromatographed (silica gel, ethyl acetate) to give the intermediate alcohol 26a (0.13 g, 64\%) as pale-yellow solid that was used for next step without an additional purification; ${ }^{1} \mathrm{H}$ NMR (400 $\left.\mathrm{MHz}, \mathrm{DMSO}-d_{6}\right) \delta 1.40(\mathrm{~s}, 9 \mathrm{H}, t-\mathrm{Bu}), 1.95-2.12\left(\mathrm{~m}, 4 \mathrm{H}, \mathrm{CH}_{2} \mathrm{CH}_{2}\right), 2.45$ (s, $\left.3 \mathrm{H}, 2-\mathrm{Me}\right), 2.81-2.92$ (m, $\left.4 \mathrm{H}, \mathrm{CH}_{2} \mathrm{CH}_{2}\right), 3.15-3.40\left(\mathrm{~m}, 2 \mathrm{H}, \mathrm{CH}_{2}\right), 3.59-3.77\left(\mathrm{~m}, 2 \mathrm{H}, \mathrm{CH}_{2}\right), 3.88-3.95(\mathrm{~m}, 1 \mathrm{H}, \mathrm{CH}), 6.52(\mathrm{~s}$, $1 \mathrm{H}, \mathrm{OH}), 8.34(\mathrm{~s}, 1 \mathrm{H}, 6-\mathrm{H})$. The alcohol 26a was dissolved in anhydrous dichloromethane $(3 \mathrm{~mL})$, and trifluoroacetic anhydride $(1 \mathrm{~mL})$ was added. The reaction mixture was stirred at room temperature for $24 \mathrm{~h}$ and concentrated under reduced pressure. The residue was dissolved in saturated aqueous solution of $\mathrm{NaHCO}_{3}(5 \mathrm{~mL})$, and the product was extracted with ethyl acetate (3 $\times 5 \mathrm{~mL})$. The extract was dried over $\mathrm{MgSO}_{4}$ and evaporated to give the titled product $25 \mathbf{b}(0.10 \mathrm{~g}$, $52 \%$, for two steps) as pale-yellow glassy mass; ${ }^{1} \mathrm{H}$ NMR $\left(400 \mathrm{MHz}, \mathrm{CDCl}_{3}\right) \delta 1.52(\mathrm{~s}, 9 \mathrm{H}, t-\mathrm{Bu})$, 2.03-2.14 (m, 2H, $\mathrm{CH}_{2}$ ), 2.55 (s, 3H, 2-Me), 2.90 (t, $\left.2 \mathrm{H}, J 7.6 \mathrm{~Hz}, \mathrm{CH}_{2}\right), 2.96$ (t, $2 \mathrm{H}, J 7.6 \mathrm{~Hz}, \mathrm{CH}_{2}$ ), 2.99 (t, $\left.2 \mathrm{H}, J 5.6 \mathrm{~Hz}, \mathrm{CH}_{2}\right), 3.82\left(\mathrm{t}, 2 \mathrm{H}, J 5.6 \mathrm{~Hz}, \mathrm{CH}_{2}\right), 4.72\left(\mathrm{~s}, 2 \mathrm{H}, \mathrm{CH}_{2}\right), 8.38(\mathrm{~s}, 1 \mathrm{H}, 6-\mathrm{H})$. APCI MS $m / z: 372[\mathrm{M}+\mathrm{H}]^{+}, 316[\mathrm{M}-t-\mathrm{Bu}+\mathrm{H}]^{+}, 272[\mathrm{M}-\mathrm{Boc}+\mathrm{H}]^{+}$. Anal. Calcd for $\mathrm{C}_{20} \mathrm{H}_{25} \mathrm{~N}_{3} \mathrm{O}_{2} \mathrm{~S}$ (371.51): C, 64.66; H, 6.78; N, 11.31; S, 8.63\%. Found: C, 64.77; H, 6.63; N, 11.05; S, 8.42\%.

4-[4-(4-Fluorophenyl)-1,3-thiazol-2-yl]-3-methyl-6,7,8,9-tetrahydro-5H-cyclohepta[c]-pyridine (25c). The mixture of thioamide 23b $(0.10 \mathrm{~g}, 0.45 \mathrm{mmol}), 2$-bromo-1-(4-fluorophenyl)ethanone 24a $(0.103 \mathrm{~g}, 0.47 \mathrm{mmol})$ and anhydrous ethanol $(1 \mathrm{~mL})$ was stirred at $75^{\circ} \mathrm{C}$ for $1 \mathrm{~h}$ then cooled, diluted with ethyl acetate $(5 \mathrm{~mL})$ and washed with saturated aqueous solution of $\mathrm{NaHCO}_{3}(5 \mathrm{~mL})$. The aqueous layer was extracted with ethyl acetate $(3 \times 10 \mathrm{~mL})$. Combined organic solutions were washed with brine $(10 \mathrm{~mL})$, dried over $\mathrm{MgSO}_{4}$, and evaporated at $60{ }^{\circ} \mathrm{C} / 20$ Torr to afford the 
product 25c (0.15 g, 99\%) as yellow glassy mass; ${ }^{1} \mathrm{H}$ NMR (400 MHz, DMSO- $\left.d_{6}\right) \delta 1.44-1.54(\mathrm{~m}$, $\left.2 \mathrm{H}, \mathrm{CH}_{2}\right), 1.55-1.64\left(\mathrm{~m}, 2 \mathrm{H}, \mathrm{CH}_{2}\right), 1.73-1.83\left(\mathrm{~m}, 2 \mathrm{H}, \mathrm{CH}_{2}\right), 2.55(\mathrm{~s}, 3 \mathrm{H}, 2-\mathrm{Me}), 2.51-2.58(\mathrm{~m}, 2 \mathrm{H}$, $\left.\mathrm{CH}_{2}\right), 2.78-2.86\left(\mathrm{~m}, 2 \mathrm{H}, \mathrm{CH}_{2}\right), 7.25-7.32\left(\mathrm{~m}, 2 \mathrm{H}, \mathrm{C}_{6} \mathrm{H}_{4}\right), 8.01-8.07\left(\mathrm{~m}, 2 \mathrm{H}, \mathrm{C}_{6} \mathrm{H}_{4}\right), 8.31(\mathrm{~s}, 1 \mathrm{H}, \mathrm{CH}$ of thiazole ring), $8.33\left(\mathrm{~s}, 1 \mathrm{H}, \mathrm{CH}\right.$ of pyridine ring). APCI MS $m / z: 339[\mathrm{M}+\mathrm{H}]^{+}$. Anal. Calcd for $\mathrm{C}_{20} \mathrm{H}_{19} \mathrm{FN}_{2} \mathrm{~S}$ (338.45): C, 70.98; H, 5.66; F, 5.61; N, 8.28; S, 9.47\%. Found: C, 71.07; H, 5.88; F, $5.42 ; \mathrm{N}, 7.99 ; \mathrm{S}, 9.29 \%$.

1-(4,5-Dimethyl-1,3-thiazol-2-yl)-2-methyl-5,6-dihydrobenzo[f]isoquinoline (25d). The mixture of thioamide $23 \mathrm{c}(0.20 \mathrm{~g}, 0.8 \mathrm{mmol})$, 3-chlorobutan-2-one $18(0.104 \mathrm{~g}, 0.98 \mathrm{mmol})$ and anhydrous ethanol $(2 \mathrm{~mL})$ was subjected to microwave irradiation at $100{ }^{\circ} \mathrm{C}$ for $4 \mathrm{~h}$. Additional 3-chlorobutan2-one 18 (0.104 g, $0.98 \mathrm{mmol})$ was added, and the microwave irradiation was continued at $100{ }^{\circ} \mathrm{C}$ for $4 \mathrm{~h}$. The reaction mixture was then concentrated under reduced pressure, and the residue was dissolved in saturated aqueous solution of $\mathrm{NaHCO}_{3}(5 \mathrm{~mL})$. The mixture was extracted with ethyl acetate $(3 \times 5 \mathrm{~mL})$; the extract was dried over $\mathrm{MgSO}_{4}$ and evaporated. The residue was chromatographed (silica gel, hexane/ethyl acetate $7: 3)$ to give the product $\mathbf{2 5 d}(0.15 \mathrm{~g}, 99 \%)$ as dark-brown oil; ${ }^{1} \mathrm{H}$ NMR (400 MHz, DMSO- $\left.d_{6}\right) \delta 2.31$ (s, 3H, Me), $2.38(\mathrm{~s}, 6 \mathrm{H}, 2 \mathrm{Me}), 2.72-2.83$ (m, 4H, $\mathrm{CH}_{2} \mathrm{CH}_{2}$ ), 6.89 (dd, 1H, J 7.7, $1.2 \mathrm{~Hz}, \mathrm{C}_{6} \mathrm{H}_{4}$ ), 7.00 (ddd, 1H, J 7.7, $1.5 \mathrm{~Hz}, \mathrm{C}_{6} \mathrm{H}_{4}$ ), 7.22 (ddd, 1H, J 7.7, $\left.1.2 \mathrm{~Hz}, \mathrm{C}_{6} \mathrm{H}_{4}\right), 7.32$ (dd, $\left.1 \mathrm{H}, J 7.7,1.5 \mathrm{~Hz}, \mathrm{C}_{6} \mathrm{H}_{4}\right), 8.48$ (s, 1H, CH of pyridine ring). APCI MS m/z: $307[\mathrm{M}+\mathrm{H}]^{+}$. Anal. Calcd for $\mathrm{C}_{19} \mathrm{H}_{18} \mathrm{~N}_{2} \mathrm{~S}$ (306.43): C, 74.47; H, 5.92; N, 9.14; S, $10.46 \%$. Found: C, 74.31; H, 5.98; N, 9.05; S, 10.22\%.

2-Methyl-4-phenyl-3-[4-(trifluoromethyl)-1,3-thiazol-2-yl]pyridine (25e). The mixture of thioamide 23d (0.10 g, $0.44 \mathrm{mmol})$, 3-bromo-1,1,1-trifluoroacetone 20 (0.092 g, $0.48 \mathrm{mmol})$ and anhydrous ethanol $(1 \mathrm{~mL})$ was stirred at $70{ }^{\circ} \mathrm{C}$ for $48 \mathrm{~h}$ then evaporated to dryness to afford the intermediate alcohol 26b (hydrobromide) (0.166 g, 90\%), that was used for next step without an additional purification; ${ }^{1} \mathrm{H} \mathrm{NMR}\left(400 \mathrm{MHz}, \mathrm{DMSO}-d_{6}\right) \delta 2.67(\mathrm{~s}, 3 \mathrm{H}, \mathrm{Me}), 3.47(\mathrm{~d}, 1 \mathrm{H}, J 13.1 \mathrm{~Hz}$, $\mathrm{CH}_{2}$ ), 3.63 (d, $1 \mathrm{H}, J 13.1 \mathrm{~Hz}, \mathrm{CH}_{2}$ ), 7.44-7.56 (m, 5H, Ph), 7.79 (d, 1H, J 5.9 Hz, 5-H), 7.88 (br. s, $2 \mathrm{H}, \mathrm{OH}, \mathrm{HBr}), 8.86(\mathrm{~d}, 1 \mathrm{H}, J 5.9 \mathrm{~Hz}, 6-\mathrm{H})$. The intermediate $\mathbf{2 6 \mathbf { b }}$ was dissolved in anhydrous dichloromethane $(5 \mathrm{~mL})$, and trifluoroacetic anhydride $(1 \mathrm{~mL})$ was added. The reaction mixture was stirred at room temperature for $24 \mathrm{~h}$ and then diluted with saturated aqueous solution of $\mathrm{NaHCO}_{3}(5$ $\mathrm{mL})$. The resulting mixture was extracted with ethyl acetate $(3 \times 10 \mathrm{~mL})$; the extract was dried over $\mathrm{MgSO}_{4}$ and evaporated to yield the target product 25e $(0.10 \mathrm{~g}, 71 \%$ for two steps $)$ as white crystal solid; mp 99-101 ${ }^{\circ} \mathrm{C} ;{ }^{1} \mathrm{H}$ NMR (400 MHz, DMSO-d $) \delta 2.42$ (s, 3H, Me), 7.14-7.21 (m, 2H, Ph), 7.29-7.35 (m, 3H, Ph), $7.40(\mathrm{~d}, 1 \mathrm{H}, J 5.1 \mathrm{~Hz}, 5-\mathrm{H}), 8.56(\mathrm{~s}, 1 \mathrm{H}, \mathrm{CH}$ of thiazole ring), $8.66(\mathrm{~d}, 1 \mathrm{H}, J$ $5.1 \mathrm{~Hz}, 6-\mathrm{H})$. APCI MS m/z: $321[\mathrm{M}+\mathrm{H}]^{+}$. Anal. Calcd for $\mathrm{C}_{16} \mathrm{H}_{11} \mathrm{~F}_{3} \mathrm{~N}_{2} \mathrm{~S}$ (320.34): C, 59.99; $\mathrm{H}$, 3.46; F, 17.79; N, 8.74; S, 10.01\%. Found: C, 60.07; H, 3.49; F, 17.60; N, 8.79; S, 9.96\%.

Ethyl 2-(2-methyl-4-phenylpyridin-3-yl)-1,3-thiazole-4-carboxylate (25f). The mixture of thioamide 23d (0.10 g, $0.44 \mathrm{mmol})$, ethyl bromopyruvate $24 \mathrm{c}(0.094 \mathrm{~g}, 0.48 \mathrm{mmol})$, and anhydrous ethanol $(1 \mathrm{~mL})$ was stirred at $70{ }^{\circ} \mathrm{C}$ for $80 \mathrm{~h}$. Additional ethyl bromopyruvate $24 \mathrm{c}(0.094 \mathrm{~g}, 0.48$ mmol) as well as $\mathrm{NaHCO}_{3}(0.11 \mathrm{~g})$ were added to the mixture that was stirred at $70{ }^{\circ} \mathrm{C}$ for $4 \mathrm{~h}$ then concentrated under reduced pressure. The residue was chromatographed (silica gel, hexane/ethyl acetate 3:1) to give the target 3-(thiazol-2-yl)pyridine $\mathbf{2 5 f}(0.018 \mathrm{~g}, 13 \%)$ and intermediate alcohol 26c $(0.11 \mathrm{~g}, 73 \%)$ that was used for next step without an additional purification; ${ }^{1} \mathrm{H}$ NMR (400 
$\left.\mathrm{MHz} \mathrm{CDCl}_{3}\right) \delta 1.35\left(\mathrm{t}, 3 \mathrm{H}, J 7.2 \mathrm{~Hz}, \mathrm{CH}_{2} \mathrm{CH}_{3}\right), 2.68(\mathrm{~s}, 3 \mathrm{H}, \mathrm{Me}), 3.40\left(\mathrm{~d}, 1 \mathrm{H}, J 12.1 \mathrm{~Hz}, \mathrm{~S}-\mathrm{CH}_{2}\right)$, $3.86\left(\mathrm{~d}, 1 \mathrm{H}, J 12.1 \mathrm{~Hz}, \mathrm{~S}-\mathrm{CH}_{2}\right), 4.29$ (br. s, $\left.1 \mathrm{H}, \mathrm{OH}\right), 4.33$ (q, $\left.2 \mathrm{H}, J 7.2 \mathrm{~Hz}, \mathrm{CH}_{2} \mathrm{CH}_{3}\right), 7.21(\mathrm{~d}, 1 \mathrm{H}, J$ $5.2 \mathrm{~Hz}, 5-\mathrm{H}), 7.37-7.43$ (m, 3H, Ph), 7.44-7.50 (m, 2H, Ph), 8.57 (d, 1H, J 5.2 Hz, 6-H). The alcohol 26c was dissolved in anhydrous dichloromethane $(2 \mathrm{~mL})$, and trifluoroacetic anhydride $(1$ $\mathrm{mL}$ ) was added. The reaction mixture was stirred at room temperature for $1 \mathrm{~h}$ and evaporated to dryness. The residue was chromatographed (silica gel, hexane/ethyl acetate 3:1) to give the desired product $25 \mathrm{f}(0.1 \mathrm{~g})$; the total yield was $0.118 \mathrm{~g}$ ( $83 \%$ for two steps), pale-yellow crystal solid; $\mathrm{mp}$ 138-140 ${ }^{\circ} \mathrm{C}$; ${ }^{1} \mathrm{H}$ NMR (400 MHz, $\left.\mathrm{CDCl}_{3}\right) \delta 1.43\left(\mathrm{t}, 3 \mathrm{H}, J 7.1 \mathrm{~Hz}, \mathrm{CH}_{2} \mathrm{CH}_{3}\right), 2.54(\mathrm{~s}, 3 \mathrm{H}, \mathrm{Me}), 4.46$ (q, 2H, J 7.1 Hz, $\left.\mathrm{CH}_{2} \mathrm{CH}_{3}\right), 7.18-7.30(\mathrm{~m}, 5 \mathrm{H}, \mathrm{Ph}), 7.28(\mathrm{~d}, 1 \mathrm{H}, J 6.1 \mathrm{~Hz}, 5-\mathrm{H}), 8.16(\mathrm{~s}, 1 \mathrm{H}, \mathrm{CH}$ of thiazole ring), $8.62(\mathrm{~d}, 1 \mathrm{H}, J 6.1 \mathrm{~Hz}, 6-\mathrm{H})$. APCI MS m/z: $325[\mathrm{M}+\mathrm{H}]^{+}$. Anal. Calcd for $\mathrm{C}_{18} \mathrm{H}_{16} \mathrm{~N}_{2} \mathrm{O}_{2} \mathrm{~S}$ (324.40): C, 66.65; H, 4.97; N, 8.64; S, 9.88\%. Found: C, 66.71; H, 5.01; N, 8.44; S, $10.02 \%$.

Ethyl [2-(2-methyl-4-phenylpyridin-3-yl)-1,3-thiazol-4-yl]acetate (25g). The mixture of thioamide 23d (0.20 g, $0.88 \mathrm{mmol})$, ethyl 4-chloro-3-oxobutanoate 24d (0.16 g, $0.97 \mathrm{mmol})$, $\mathrm{NaHCO}_{3}(0.15 \mathrm{~g}, 1.76 \mathrm{mmol})$, and anhydrous ethanol $(2 \mathrm{~mL})$ was stirred at $75{ }^{\circ} \mathrm{C}$ for $3 \mathrm{~h}$ then cooled, diluted with ethyl acetate $(10 \mathrm{~mL})$ and filtered. The filtrate was concentrated under reduced pressure to give the intermediate alcohol 26d (0.32 g, 100\%, with impurities) as pale-yellow oil that was used for next step without an additional purification; ${ }^{1} \mathrm{H} \mathrm{NMR}\left(400 \mathrm{MHz}, \mathrm{CDCl}_{3}\right) \delta 1.30(\mathrm{t}, 3 \mathrm{H}$, $\left.J 7.3 \mathrm{~Hz}, \mathrm{CH}_{2} \mathrm{CH}_{3}\right), 2.67$ (s, 3H, Me), $2.73\left(\mathrm{~s}, 1 \mathrm{H}, \mathrm{CH}_{2}\right), 2.74\left(\mathrm{~s}, 1 \mathrm{H}, \mathrm{CH}_{2}\right), 3.30$ (d, $1 \mathrm{H}, J 12.0 \mathrm{~Hz}$, S-CH $)_{2}, 3.41\left(\mathrm{~d}, 1 \mathrm{H}, J 12.0 \mathrm{~Hz}, \mathrm{~S}-\mathrm{CH}_{2}\right), 4.23$ (q, $2 \mathrm{H}, J 7.3 \mathrm{~Hz}, \mathrm{CH}_{2} \mathrm{CH}_{3}$ ), 4.89 (br. s, $\left.1 \mathrm{H}, \mathrm{OH}\right), 7.17$ (d, 1H, J 5.1 Hz, 5-H), 7.38-7.46 (m, 5H, Ph), 8.56 (d, 1H, J 5.1 Hz, 6-H). The alcohol 26d was dissolved in anhydrous dichloromethane $(2 \mathrm{~mL})$, and trifluoroacetic anhydride $(1 \mathrm{~mL})$ was added. The reaction mixture was stirred at room temperature for $12 \mathrm{~h}$ and evaporated to dryness. The residue was dissolved in saturated aqueous solution of $\mathrm{NaHCO}_{3}(5 \mathrm{~mL})$, and the solution was treated with ethyl acetate $(3 \times 5 \mathrm{~mL})$. The extract was dried over $\mathrm{MgSO}_{4}$ and concentrated under reduced pressure. The residue was chromatographed (silica gel, hexane/ethyl acetate 7:3) to give the product 25g $\left(0.26 \mathrm{~g}, 88 \%\right.$ ) as brown solid; mp 78-80 ${ }^{\circ} \mathrm{C} ;{ }^{1} \mathrm{H} \mathrm{NMR}\left(400 \mathrm{MHz}, \mathrm{CDCl}_{3}\right) \delta 1.29(\mathrm{t}, 3 \mathrm{H}, J 7.2$ $\mathrm{Hz}, \mathrm{CH}_{2} \mathrm{CH}_{3}$ ), 2.52 (s, 3H, Me), 3.87 (s, 2H, $\mathrm{CH}_{2}$ ), 4.21 (q, 2H, J 7.2 Hz, $\left.\mathrm{CH}_{2} \mathrm{CH}_{3}\right), 7.17$ (s, 1H, CH of thiazole ring), 7.18-7.22 (m, 2H, Ph), 7.23 (d, 1H, J 5.3 Hz, 5-H), 7.24-7.29 (m, 3H, Ph), 8.60 (d, $1 \mathrm{H}, J 5.3 \mathrm{~Hz}, 6-\mathrm{H})$. APCI MS $m / z: 339[\mathrm{M}+\mathrm{H}]^{+}$. Anal. Calcd for $\mathrm{C}_{19} \mathrm{H}_{18} \mathrm{~N}_{2} \mathrm{O}_{2} \mathrm{~S}$ (338.43): C, 67.43; H, 5.36; N, 8.28; S, 9.47\%. Found: C, 67.34; H, 5.36; N, 8.24; S, 9.41\%.

Ethyl 4-methyl-2-(2-methyl-4-phenylpyridin-3-yl)-1,3-thiazole-5-carboxylate (25h). The mixture of thioamide $23 \mathbf{d}(0.111 \mathrm{~g}, 0.49 \mathrm{mmol})$, ethyl 2-chloro-3-oxobutanoate $24 \mathrm{e}(0.084 \mathrm{~g}, 0.51$ $\mathrm{mmol})$, and anhydrous ethanol $(1 \mathrm{~mL})$ was stirred at $50{ }^{\circ} \mathrm{C}$ for $50 \mathrm{~h}$ then concentrated under reduced pressure to afford hydrochloride of the intermediate alcohol $26 \mathrm{e}(0.14 \mathrm{~g}, 72 \%)$ as yellow solid that was used for next step without an additional purification $\left({ }^{1} \mathrm{H}\right.$ NMR spectra in DMSO- $d_{6}$ was an intricate superposition of two spectra for two diastereomers). The alcohol 26e was dissolved in anhydrous dichloromethane $(3 \mathrm{~mL})$, and trifluoroacetic anhydride $(0.5 \mathrm{~mL})$ was added. The reaction mixture was stirred at room temperature for $3 \mathrm{~h}$ and evaporated to dryness. The residue was dissolved in saturated aqueous solution of $\mathrm{NaHCO}_{3}(5 \mathrm{~mL})$, and the solution was extracted with ethyl acetate $(3 \times 5 \mathrm{~mL})$. The extract was dried over $\mathrm{MgSO}_{4}$ and concentrated under reduced 
pressure. The residue was chromatographed (silica gel, hexane/ethyl acetate 3:1) to give the desired product $25 \mathrm{~h}(0.112 \mathrm{~g}, 68 \%)$ as pale-yellow powder; mp $104-105{ }^{\circ} \mathrm{C} ;{ }^{1} \mathrm{H} \mathrm{NMR}\left(400 \mathrm{MHz}, \mathrm{CDCl}_{3}\right) \delta$ $1.32\left(\mathrm{t}, 3 \mathrm{H}, J 7.1 \mathrm{~Hz}, \mathrm{CH}_{2} \mathrm{CH}_{3}\right), 2.52(\mathrm{~s}, 3 \mathrm{H}, \mathrm{Me}), 2.76(\mathrm{~s}, 3 \mathrm{H}, \mathrm{Me}), 4.28\left(\mathrm{q}, 2 \mathrm{H}, J 7.1 \mathrm{~Hz}, \mathrm{CH}_{2} \mathrm{CH}_{3}\right)$, 7.17-7.24 (m, 3H, Ph), 7.27-7.31 (m, 2H, Ph), 7.29 (d, 1H, J 5.1 Hz, 5-H), 8.61 (d, 1H, J 5.1 Hz, 6H). APCI MS m/z: $339[\mathrm{M}+\mathrm{H}]^{+}$. Anal. Calcd for $\mathrm{C}_{19} \mathrm{H}_{18} \mathrm{~N}_{2} \mathrm{O}_{2} \mathrm{~S}$ (338.43): C, 67.43; H, 5.36; N, 8.28; S, 9.47\%. Found: C, 67.39; H, 5.47; N, 8.31; S, 9.64\%.

\section{4-tert-Butyl-3-(4-ethyl-1,3-thiazol-2-yl)-2-methylpyridine (25i)}

Method A. The mixture of 4-tert-butyl-2-methylpyridine-3-carbothioamide 23e $(0.11 \mathrm{~g}, 0.49$ mmol), 1-bromobutan-2-one $24 \mathbf{f}(0.09 \mathrm{~g}, 0.59 \mathrm{mmol})$, and anhydrous ethanol $(1 \mathrm{~mL})$ was stirred at room temperature for $24 \mathrm{~h}$ then concentrated at $60{ }^{\circ} \mathrm{C} / 20$ Torr. The residue was mixed with ethyl acetate $(5 \mathrm{~mL})$. The precipitate was filtered off, washed with ether and dissolved in saturated aqueous solution of $\mathrm{NaHCO}_{3}(5 \mathrm{~mL})$, and the solution was extracted with ethyl acetate $(3 \times 5 \mathrm{~mL})$. The extract was dried over $\mathrm{MgSO}_{4}$ and concentrated under reduced pressure to give the intermediate alcohol $\mathbf{2 6 f}(0.13 \mathrm{~g}, 90 \%)$ as pale-yellow oil that was used for next step without an additional purification; ${ }^{1} \mathrm{H}$ NMR (400 MHz, DMSO- $\left.d_{6}\right) \delta 1.00\left(\mathrm{t}, 3 \mathrm{H}, J 7.0 \mathrm{~Hz}, \mathrm{CH}_{2} \mathrm{CH}_{3}\right), 1.40(\mathrm{~s}$, $9 \mathrm{H}, t$-Bu), 1.90 (q, 2H, J 7.0 Hz, $\mathrm{CH}_{2} \mathrm{CH}_{3}$ ), 2.45 (s, 3H, Me), 3.22-3.38 (m, 1H, S-CH $), 3.44-3.59$ (m, 1H, S-CH2), 6.06 (br. s, 1H, OH), 7.31 (d, 1H, J $5.4 \mathrm{~Hz}, 5-\mathrm{H}), 8.38$ (d, 1H, J 5.4 Hz, 6-H). The alcohol $26 \mathbf{f}$ was dissolved in anhydrous 1,2-dichloroethane $(5 \mathrm{~mL})$, and trifluoroacetic anhydride (1 $\mathrm{mL}$ ) was added. The reaction mixture was stirred at room temperature for $1 \mathrm{~h}$ and evaporated to dryness. The residue was dissolved in saturated aqueous solution of $\mathrm{NaHCO}_{3}(5 \mathrm{~mL})$, and the solution was extracted with ethyl acetate $(3 \times 10 \mathrm{~mL})$. The extract was dried over $\mathrm{MgSO}_{4}$ and concentrated under reduced pressure to give the titled product $\mathbf{2 5 i}(0.098 \mathrm{~g}, 71 \%)$ as pale-yellow oil; ${ }^{1} \mathrm{H} \mathrm{NMR}\left(400 \mathrm{MHz}, \mathrm{CDCl}_{3}\right) \delta 1.19(\mathrm{~s}, 9 \mathrm{H}, t-\mathrm{Bu}), 1.34\left(\mathrm{t}, 3 \mathrm{H}, J 7.3 \mathrm{~Hz}, \mathrm{CH}_{2} \mathrm{CH}_{3}\right), 2.24(\mathrm{~s}, 3 \mathrm{H}, \mathrm{Me})$, 2.88 (q, 2H, J 7.3 Hz, $\left.\mathrm{CH}_{2} \mathrm{CH}_{3}\right), 7.07$ (s, $1 \mathrm{H}, \mathrm{CH}$ of thiazole ring), 7.28 (d, 1H, J 5.6 Hz, 5-H), 7.44 (d, $1 \mathrm{H}, J 5.6 \mathrm{~Hz}, 6-\mathrm{H})$. APCI MS $m / z: 261[\mathrm{M}+\mathrm{H}]^{+}$. Anal. Calcd for $\mathrm{C}_{15} \mathrm{H}_{20} \mathrm{~N}_{2} \mathrm{~S}$ (260.40): C, 69.19; H, 7.74; N, 10.76; S, 12.31\%. Found: C, 69.24; H, 7.83; N, 10.59; S, 12.22\%.

Method B. The mixture of 4-tert-butyl-2-methylpyridine-3-carbothioamide 23e $(0.1 \mathrm{~g}, 0.48 \mathrm{mmol})$, 1-bromobutan-2-one $24 \mathrm{f}(0.09 \mathrm{~g}, 0.59 \mathrm{mmol})$, and anhydrous ethanol $(1 \mathrm{~mL})$ was stirred at $75{ }^{\circ} \mathrm{C}$ for $5 \mathrm{~h}$ then concentrated at $60{ }^{\circ} \mathrm{C} / 20$ Torr. The residue was mixed with ethyl acetate $(5 \mathrm{~mL})$. The precipitate was filtered off, washed with ether and dried to give the target product (hydrobromide) 25i (0.095 g, 58\%) as pale-yellow solid; mp 175-176 ${ }^{\circ} \mathrm{C} ;{ }^{1} \mathrm{H}$ NMR (400 MHz, DMSO- $\left.d_{6}\right) \delta 1.20(\mathrm{~s}$, $9 \mathrm{H}, t-\mathrm{Bu}), 1.26\left(\mathrm{t}, 3 \mathrm{H}, J 7.3 \mathrm{~Hz}, \mathrm{CH}_{2} \mathrm{CH}_{3}\right), 2.33$ (s, 3H, Me), 2.82 (q, 2H, J 7.3 Hz, $\left.\mathrm{CH}_{2} \mathrm{CH}_{3}\right), 7.67$ (s, $1 \mathrm{H}, \mathrm{CH}$ of thiazole ring), $8.05(\mathrm{~d}, 1 \mathrm{H}, J 5.6 \mathrm{~Hz}, 5-\mathrm{H}), 8.83(\mathrm{~d}, 1 \mathrm{H}, J 5.6 \mathrm{~Hz}, 6-\mathrm{H})$. APCI MS $m / z$ : $261[\mathrm{M}+\mathrm{H}]^{+}$. Anal. Calcd for $\mathrm{C}_{15} \mathrm{H}_{20} \mathrm{~N}_{2} \mathrm{~S} \times 1.1 \mathrm{HBr}$ (349.41): C, 51.56; H, 6.09; Br, 25.16; N, 8.02; S, 9.18\%. Found: C, 51.33; H, 6.22; Br, 24.93; N, 7.87; S, 8.97\%.

4-Isopropyl-2-methyl-3-[4-(4-methylphenyl)-1,3-thiazol-2-yl]pyridine hydrobromide (25j). The mixture of 4-isopropyl-2-methylpyridine-3-carbothioamide $23 f \quad(0.1 \quad \mathrm{~g}, 0.52 \mathrm{mmol})$, 4methylphenacyl bromide $\mathbf{2 4 g}(0.115 \mathrm{~g}, 0.54 \mathrm{mmol})$, and anhydrous ethanol $(1 \mathrm{~mL})$ was stirred at 75 ${ }^{\circ} \mathrm{C}$ for $80 \mathrm{~min}$ then concentrated at $60{ }^{\circ} \mathrm{C} / 20$ Torr. The residue was mixed with ethyl acetate $(5 \mathrm{~mL})$. The precipitate was filtered off, washed with ether and dried to give the target product $25 \mathbf{j}(0.18 \mathrm{~g}$, $90 \%$ ) as white crystal solid; mp $186-187{ }^{\circ} \mathrm{C} ;{ }^{1} \mathrm{H}$ NMR (400 MHz, DMSO- $\left.d_{6}\right) \delta 1.22$ (d, 6H, $J 6.8$ 
$\left.\mathrm{Hz}, \mathrm{CH}\left(\mathrm{CH}_{3}\right)_{2}\right), 2.35$ (s, 3H, Me), 2.50 (s, 3H, Me), 2.81-2.90 (m, 1H, $\left.\mathrm{CH}\left(\mathrm{CH}_{3}\right)_{2}\right), 7.28$ (d, $2 \mathrm{H}, J 8.0$ $\left.\mathrm{Hz}, \mathrm{C}_{6} \mathrm{H}_{4}\right), 7.89$ (d, 2H, J $\left.8.0 \mathrm{~Hz}, \mathrm{C}_{6} \mathrm{H}_{4}\right), 8.00(\mathrm{~d}, 1 \mathrm{H}, J 6.1 \mathrm{~Hz}, 5-\mathrm{H}), 8.42(\mathrm{~s}, 1 \mathrm{H}, \mathrm{CH}$ of thiazole ring), $8.87(\mathrm{~d}, 1 \mathrm{H}, J 6.1 \mathrm{~Hz}, 6-\mathrm{H})$. APCI MS m/z: $309[\mathrm{M}+\mathrm{H}]^{+}$. Anal. Calcd for $\mathrm{C}_{19} \mathrm{H}_{21} \mathrm{BrN}_{2} \mathrm{~S}$ (389.36): C, 58.61; H, 5.44; Br, 20.52; N, 7.19; S, 8.24\%. Found: C, 58.54; H, 5.41; Br, 20.39; N, $7.11 ; \mathrm{S}, 8.17 \%$.

3-Methyl-4-[4-(4-methylphenyl)-1,3-thiazol-2-yl]-5,6,7,8-tetrahydroisoquinoline hydrobromide (25k). Prepared analogously from 3-methyl-5,6,7,8-tetrahydroisoquinoline-4-carbothioamide $\mathbf{2 3 g}$ (0.1 g, $0.49 \mathrm{mmol})$ and 4-methylphenacyl bromide $24 \mathrm{~g}(0.11 \mathrm{~g}, 0.51 \mathrm{mmol})$. Yield $0.156 \mathrm{~g}(80 \%)$; white crystal solid; mp 277-278 ${ }^{\circ} \mathrm{C}$ (decomp.); ${ }^{1} \mathrm{H}$ NMR (400 MHz, DMSO- $\left.d_{6}\right) \delta 1.68-1.82(\mathrm{~m}, 4 \mathrm{H}$, $\mathrm{CH}_{2} \mathrm{CH}_{2}$ ), 2.35 (s, 3H, Me), 2.47 (s, 3H, Me), 2.62-2.72 (m, 2H, $\left.\mathrm{CH}_{2}\right), 2.87-2.97$ (m, 2H, $\left.\mathrm{CH}_{2}\right), 7.28$ $\left(\mathrm{d}, 2 \mathrm{H}, J 8.1 \mathrm{~Hz}, \mathrm{C}_{6} \mathrm{H}_{4}\right), 7.88\left(\mathrm{~d}, 2 \mathrm{H}, J 8.1 \mathrm{~Hz}, \mathrm{C}_{6} \mathrm{H}_{4}\right), 8.41(\mathrm{~s}, 1 \mathrm{H}, \mathrm{CH}$ of thiazole ring), $8.72(\mathrm{~s}, 1 \mathrm{H}$, $\mathrm{CH}$ of pyridine ring). APCI MS m/z: $321[\mathrm{M}+\mathrm{H}]^{+}$. Anal. Calcd for $\mathrm{C}_{20} \mathrm{H}_{21} \mathrm{BrN}_{2} \mathrm{~S}$ (401.37): C, 59.85; H, 5.27; Br, 19.91; N, 6.98; S, 7.99\%. Found: C, 59.92; H, 5.31; Br, 19.79; N, 7.04; S, 7.90\%.

\section{4-[4-(3-Methoxyphenyl)-1,3-thiazol-2-yl]-3,5-dimethyl-5,6,7,8-tetrahydroisoquinoline}

hydrobromide (25l). Prepared analogously from 3,5-dimethyl-5,6,7,8-tetrahydroisoquinoline-4carbothioamide $23 \mathbf{h}(0.1 \mathrm{~g}, 0.45 \mathrm{mmol})$ and 3-methoxylphenacyl bromide $24 \mathrm{~h}(0.11 \mathrm{~g}, 0.48 \mathrm{mmol})$, heating at $70{ }^{\circ} \mathrm{C}$ for $2 \mathrm{~h}$. Yield $0.192 \mathrm{~g}$ (98\%); white crystal solid; mp 235-236 ${ }^{\circ} \mathrm{C}$ (decomp.); ${ }^{1} \mathrm{H}$ NMR (400 MHz, DMSO- $\left.d_{6}\right) \delta 1.06\left(\mathrm{~d}, 3 \mathrm{H}, J 7.3 \mathrm{~Hz}, \mathrm{CH}-\mathrm{CH}_{3}\right), 1.64-1.94\left(\mathrm{~m}, 4 \mathrm{H}, \mathrm{CH}_{2} \mathrm{CH}_{2}\right), 2.45$ (s, 3H, Me), 2.80-2.94 (m, 1H, CH-CH $), 3.00-3.21\left(\mathrm{~m}, 2 \mathrm{H}, \mathrm{CH}_{2}\right), 3.82(\mathrm{~s}, 3 \mathrm{H}, \mathrm{OMe}), 6.97$ (ddd, $1 \mathrm{H}, J$ 8.2, 2.7, $\left.1.0 \mathrm{~Hz}, 4-\mathrm{H}, \mathrm{C}_{6} \mathrm{H}_{4}\right), 7.39$ (dd, 1H, J 8.2, 7.8 Hz, 5-H, $\left.\mathrm{C}_{6} \mathrm{H}_{4}\right), 7.55$ (dd, 1H, J 2.7, 1.0 $\left.\mathrm{Hz}, 2-\mathrm{H}, \mathrm{C}_{6} \mathrm{H}_{4}\right), 7.59$ (ddd, $1 \mathrm{H}, J 7.8,1.0,1.0 \mathrm{~Hz}, 6-\mathrm{H}, \mathrm{C}_{6} \mathrm{H}_{4}$ ), 8.56 (s, 1H, CH of thiazole ring), $8.76\left(\mathrm{~s}, 1 \mathrm{H}, \mathrm{CH}\right.$ of pyridine ring). APCI MS m/z: $351[\mathrm{M}+\mathrm{H}]^{+}$. Anal. Calcd for $\mathrm{C}_{21} \mathrm{H}_{23} \mathrm{BrN}_{2} \mathrm{OS}$ (431.40): C, 58.47; H, 5.37; Br, 18.52; N, 6.49; S, 7.43\%. Found: C, 58.50; H, 5.40; Br, 18.43; N, $6.36 ; \mathrm{S}, 7.25 \%$.

1-(4-tert-Butyl-1,3-thiazol-2-yl)-2-methyl-5H-chromeno[3,4-c]pyridine (25m). The mixture of 2methyl-5H-chromeno[3,4-c]pyridine-1-carbothioamide 23i (0.10 g, $0.39 \mathrm{mmol})$, 1-bromo-3,3dimethylbutan-2-one $24 \mathbf{i}(0.077 \mathrm{~g}, 0.43 \mathrm{mmol})$ and anhydrous ethanol $(1 \mathrm{~mL})$ was stirred at $70{ }^{\circ} \mathrm{C}$ for $1 \mathrm{~h}$ then concentrated under reduced pressure. The residue was diluted in saturated aqueous solution of $\mathrm{NaHCO}_{3}(5 \mathrm{~mL})$. The mixture was extracted with ethyl acetate $(3 \times 5 \mathrm{~mL})$. The extract was dried over $\mathrm{MgSO}_{4}$ and evaporated at $60{ }^{\circ} \mathrm{C} / 20$ Torr. The residue was chromatographed (silica gel, hexane/ethyl acetate 7:3) to give the target product $25 \mathrm{~m}(0.06 \mathrm{~g}, 46 \%)$ as pale-yellow glassy mass; ${ }^{1} \mathrm{H}$ NMR (400 MHz, DMSO-d 6$) \delta 1.37$ (s, 9H, $t$-Bu), $2.30(\mathrm{~s}, 3 \mathrm{H}, \mathrm{Me}), 5.13\left(\mathrm{~s}, 2 \mathrm{H}, \mathrm{CH}_{2}\right)$, 6.50 (dd, 1H, J 8.1, 1.5 Hz, $\left.\mathrm{C}_{6} \mathrm{H}_{4}\right), 6.72$ (ddd, $1 \mathrm{H}, J$ 8.1, 7.3, $\left.1.2 \mathrm{~Hz}, \mathrm{C}_{6} \mathrm{H}_{4}\right), 7.05$ (dd, 1H, J 8.1, 1.2 $\left.\mathrm{Hz}, \mathrm{C}_{6} \mathrm{H}_{4}\right), 7.27$ (ddd, $1 \mathrm{H}, J$ 8.1, 7.3, $\left.1.5 \mathrm{~Hz}, \mathrm{C}_{6} \mathrm{H}_{4}\right), 7.54(\mathrm{~s}, 1 \mathrm{H}, \mathrm{CH}$ of thiazole ring), $8.54(\mathrm{~s}, 1 \mathrm{H}$, $\mathrm{CH}$ of pyridine ring). APCI MS $m / z: 337[\mathrm{M}+\mathrm{H}]^{+}$. Anal. Calcd for $\mathrm{C}_{20} \mathrm{H}_{20} \mathrm{~N}_{2} \mathrm{OS}$ (336.46): C, 71.40; H, 5.99; N, 8.33; S, 9.53\%. Found: C, 71.31; H, 5.98; N, 8.29; S, 9.35\%.

\section{References and Notes}

1. Thornber, C. W. Chem. Soc. Rev. 1979, 8, 563. 
2. Moltzen, E. K.; Pedersen, H.; Bøgesø, K. P.; Meier, E.; Frederiksen, K.; Sánchez, C.; Lembøl, H. L. J. Med. Chem. 1994, 37, 4085.

3. Herr, R. J. Bioorg. Med. Chem. 2002, 10, 3379 and references cited therein.

4. Butler, R. N.; Garvin, V. C. J. Chem. Research (S) 1982, 122.

5. Holland, G. F.; Pereira, J. N. J. Med. Chem. 1967, 10, 149.

6. Pachhamia, V. L.; Parikh, A. R. J. Indian Chem. Soc. 1989, 66, 250.

7. Ohmoto, K.; Yamamoto, T.; Okuma, M.; Horiuchi, T.; Imanishi, H.; Odagaki, Y.; Kawabata, K.; Sekioka, T.; Hirota, Y.; Matsuoka, S.; Nakai, H.; Toda, M.; Cheronis, J. C.; Spruce, L. W.; Gyorkos, A.; Wieczorek, M. J. Med. Chem. 2001, 44, 1268.

8. (a) Showell, G. A.; Gibbons, T. L.; Kneen, C. O.; MacLeod, A. M.; Merchant, K.; Saunders, J.; Freedman, S. B.; Patel, S.; Baker, R. J. Med. Chem. 1991, 34, 1086. (b) Diana, G. D.; Volkots, D. L.; Nitz, T. J.; Bailey, T. R.; Long, M. A.; Vescio, N.; Aldous, S.; Pevear, D. C.; Dutko, F. J. J. Med. Chem. 1994, 37, 2421. (c) Messer, W. S.; Abuh, Y. F.; Liu, Y.; Periyasamy, S.; Ngur, D. O.; Edgar, M. A. N.; El-Assadi, A. A.; Sbeih, S.; Dunbar, P. G.; Roknich, S.; Rho, T.; Fang, Z.; Ojo, B.; Zhang, H.; Huzl, J. J.; Nagy, P. I. J. Med. Chem. 1997, 40, 1230. (d) Poulain, R. F.; Tartar, A. L.; Deprez, B. P. Tetrahedron Lett. 2001, 42, 1495 and references cited therein.

9. Gezginci, M. H.; Martin, A. R.; Franzblau, S. G. J. Med. Chem. 2001, 44, 1560 and referenced cited therein.

10. Ainsworth, C.; Buting, W. E.; Davenport, J.; Callender, M. E.; McCowen, M. C. J. Med. Chem. 1967, 10, 208.

11. Baldwin, J. J.; Lumma, P. K.; Novello, F. C.; Ponticello, G. S.; Sprague, J. M.; Duggan, D. E. J. Med. Chem. 1977, 20, 1189-1193.

12. Khanna, I. K.; Yu, Y.; Huff, R. M.; Weier, R. M.; Xu, X.; Koszyk, F. J.; Collins, P. W.; Cogburn. J. N.; Isakson, P. C.; Koboldt, C. M.; Masferrer, J. L.; Perkins, W. E.; Seibert, K.; Veenhuizen, A. W.; Yuan, J.; Yang, D.-C.; Zhang, Y. Y. J. Med. Chem. 2000, 43, 3168.

13. Elliott, R. L.; Oliver, R. M.; Hammond, M.; Patterson, T. A.; She, L.; Hargrove, D. M.; Martin, K. A.; Maurer, T. S.; Kalvass, J. C.; Morgan, B. P.; DaSilva-Jardine, P. A.; Stevenson, R. W.; Mack, C. M.; Cassella, J. V. J. Med. Chem. 2003, 46, 670.

14. Manley, P. J.; Balitza, A. E.; Bilodeau, M. T.; Coll, K. E.; Hartman, G. D.; McFall, R. C.; Rickert, K. W.; Rodman, L. D.; Thomas, K. A. Bioorg. Med. Chem. Lett. 2003, 13, 1673.

15. Chihiro, M.; Nagamoto, H.; Takemura, I.; Kitano, K.; Komatsu, H.; Sekiguchi, K.; Tabusa, F.; Mori, T.; Tominaga, M.; Yabuuchi, Y. J. Med. Chem. 1995, 38, 353.

16. Carter, J. S.; Rogier, D. J.; Graneto, M. J.; Seibert, K.; Koboldt, C. M.; Zhang, Y.; Talley, J. J. Bioorg. Med. Chem. Lett. 1999, 9, 1167.

17. Denton, T. T.; Zhang, X.; Cashman, J. R. J. Med. Chem. 2005, 48, 224.

18. Ingle, R. D.; Bhingolikar, V. E.; Bondge, S. P; Mane, R. A. Ind. J. Chem. 2003, 42B, 695.

19. Khadse, B. G.; Lokhande, S. R.; Bhamaria, R. P.; Prabhu, S. R. Ind. J. Chem. 1987, 26B, 856.

20. Kelly, T. R.; Echavarren, A.; Chandrakumar, N. S.; Köksal, Y. Tetrahedron Lett. 1984, 25, 2127. 
21. (a) Kelly, T. R.; Jagoe, C. T.; Gu, Z. Tetrahedron Lett. 1991, 32, 4263. (b) Ciufolini, M. A.; Shen, Y. C. J. Org. Chem. 1997, 62, 3804. (c) Okumura, K.; Shigekuni, M.; Nakamura, Y.; Shin, C. Chem. Lett. 1996, 1025.

22. Umemura, K.; Ikeda, S.; Yoshimura, J.; Okumura, K.; Saito, H.; Shin, C. Chem. Lett. 1997, 1203.

23. Okumura, K.; Suzuki, T.; Shin, C. Heterocycles 2000, 53, 765.

24. Shin, C.; Saito, H.; Yonezawa, Y. Heterocycles 2003, 61, 45.

25. (a) Duncia, J. V.; Pierce, M. E.; Santella III, J. B. J. Org. Chem. 1991, 56, 2395. (b) Wittenberger, S. J.; Donner, B. G. J. Org. Chem. 1993, 58, 4139.

26. Sams, C. K.; Lau, J. Tetrahedron Lett. 1999, 40, 9359.

27. Lukyanov, S. M.; Bliznets, I. V.; Shorshnev, S. V.; Aleksandrov, G. G.; Stepanov, A. E.; Vasil'ev, A. A. Tetrahedron 2006, 62, 1849.

28. (a) Bliznets, I. V.; Vasil'ev, A. A.; Shorshnev, S. V.; Stepanov, A. E.; Lukyanov, S. M. Tetrahedron Lett. 2004, 45, 2571. (b) Bliznets, I. V.; Shorshnev, S. V.; Aleksandrov, G. G.; Stepanov, A. E.; Lukyanov, S. M. Tetrahedron Lett. 2004, 45, 9127.

29. Hemming, K. In: Science of Synthesis, Houben-Weyl Methods of Molecular Transformations; Storr, R. C., Gilchrist, T. L., Eds.; Georg Thieme Verlag: Stuttgart, New York, 2006; Vol. 13, p 127.

30. Perez, M. A.; Dorado, C. A.; Soto, J. L. Synthesis 1983, 483.

31. (a) Watjen, F.; Baker, R.; Engelstoff, M.; Herbert, R.; MacLeod, A.; Knight, A.; Merchant, K.; Moseley, J.; Saunders, J.; Swain, C. J.; Wong, E.; Springer, J. P. J. Med. Chem. 1989, 32, 2282. (b) Ellingboe, J. W.; Alessi, T. R.; Dolak, T. M.; Nguyen, T. T.; Tomer, J. D.; Guzzo, F.; Bagli, J. F.; McCaleb, M. L. J. Med. Chem. 1992, 35, 1176. (c) Andersen, K. E.; Jørgensen, A. S.; Bræstrup, C. Eur. J. Med. Chem. 1994, 29, 393. (d) Judkins, B. D.; Allen, D. G.; Cook, T. A.; Evans, B.; Sardharwala, T. E. Synth. Commun. 1996, 26, 4351.

32. Deegan, T. L.; Nitz, T. J.; Cebzanov, D.; Pufko, D. E.; Porco, J. A. Bioorg. Med. Chem. Lett. 1999, 9, 209.

33. (a) Schaefer, F. C.; Peters, G. A. J. Org. Chem. 1961, 26, 412. (b) Leschke, C.; Elz, S.; Garbarg, M.; Schunack, W. J. Med. Chem. 1995, 38, 1287.

34. Stephenson, L.; Warburton, W. K.; Wilson, M. J. J. Chem. Soc. (C) 1969, 861.

35. Gangloff, A. R.; Litvak, J.; Shelton, E. J.; Sperandio, D.; Wang, V. R.; Rice, K. D. Tetrahedron Lett. 2001, 42, 1441.

36. Eynde, J. J. V.; Mayence, A.; Lecour, L.; Huang, T. L. Med. Chem. Res. 2003, 12, 401.

37. (a) Ogonor, J. I. Tetrahedron 1981, 37, 2909. (b) Marsura, A.; Luu-Duc, C.; Gellon, G. Synthesis 1985, 537.

38. Elliott, R. L.; Oliver, R. M.; LaFlamme, J. A.; Gillaspy, M. L.; Hammond, M.; Hank, R. F.; Maurer, T. S.; Baker, D. L.; DaSilva-Jardine, P. A.; Stevenson, R. W.; Mack, C. M.; Cassella, J. V. Bioorg. Med. Chem. Lett. 2003, 13, 3593.

39. Lange, U. E. W.; Schäfer, B.; Baucke, D.; Buschmann, E.; Mack, H. Tetrahedron Lett. 1999, 40, 7067. 
40. (a) Garigipati, R. S. Tetrahedron Lett. 1990, 31, 1969. (b) Moss, R. A.; Ma, W.; Merrer, D. C.; Xue, S. Tetrahedron Lett. 1995, 36, 8761. (c) Khanna, I. K.; Weier, R. M.; Yu, Y.; Xu, X. D.; Koszyk, F. J.; Collins, P. W.; Koboldt, C. M.; Veenhuizen, A. W.; Perkins, W. E.; Casler, J. J.; Masferrer, J. L.; Zhang, Y. Y.; Gregory, S. A.; Seibert, K.; Isakson, P. C. J. Med. Chem. 1997, 40, 1634.

41. (a) Shabana, R.; Meyer, H.J.; Lawesson, S.-O. Phosphorus Sulfur 1985, 25, 297. (b) Benner, S. A. Tetrahedron Lett. 1981, 22, 1851.

42. Bagley, M. C.; Chapaneri, K.; Glover, C.; Merritt, E. A. Synlett 2004, 2615.

43. Junghahn, A.; Bunimowicz, J. Chem. Ber. 1902, 35, 3932.

44. Raffo, M.; Rossi, G. Gazz. Chim. Ital. 1914, 44, I, 104.

45. Dondoni, A.; Merino, P. In: Comprehensive Heterocyclic Chemistry II; Katritzky, A. R., Rees, C. W., Scriven, E. F. V., Eds., Pergamon: Oxford, 1996; Vol. 3, p 373.

46. Schmidt, U.; Gleich, P.; Griesser, H.; Utz, R. Synthesis 1986, 992.

47. (a) Poroikov, V. V.; Filimonov, D. A.; Ihlenfeldt, W. D.; Gloriozova, T. A.; Lagunin, A. A.; Borodina, Yu. V.; Stepanchikova, A. V.; Nicklaus, M. C. J. Chem. Inf. Comput. Sci. 2003, 43, 228; (b) Stepanchikova, A. V.; Lagunin, A. A.; Filimonov, D. A.; Poroikov, V. V. Curr. Med. Chem. 2003, 10, 225.

48. Katritzky, A. R.; Denisenko, A.; Arend, M. J. Org. Chem. 1999, 64, 6076.

49. Ungnade, H. E.; Kissinger, L. W. J. Org. Chem. 1958, 23, 1794. 